\title{
Effects of Acute and Repeated Administration of Diazepam on Delay Discounting in Lewis and Fischer 344 Rats
}

\author{
Sally L. Huskinson \\ West Virginia University
}

Follow this and additional works at: https://researchrepository.wvu.edu/etd

\section{Recommended Citation}

Huskinson, Sally L., "Effects of Acute and Repeated Administration of Diazepam on Delay Discounting in Lewis and Fischer 344 Rats" (2011). Graduate Theses, Dissertations, and Problem Reports. 4733.

https://researchrepository.wvu.edu/etd/4733

This Thesis is protected by copyright and/or related rights. It has been brought to you by the The Research Repository @ WVU with permission from the rights-holder(s). You are free to use this Thesis in any way that is permitted by the copyright and related rights legislation that applies to your use. For other uses you must obtain permission from the rights-holder(s) directly, unless additional rights are indicated by a Creative Commons license in the record and/ or on the work itself. This Thesis has been accepted for inclusion in WVU Graduate Theses, Dissertations, and Problem Reports collection by an authorized administrator of The Research Repository @ WVU. For more information, please contact researchrepository@mail.wvu.edu. 


\title{
Effects of Acute and Repeated Administration of Diazepam on \\ Delay Discounting in Lewis and Fischer 344 Rats
}

\section{Sally L. Huskinson}

\author{
Thesis submitted to the Eberly College of Arts and Sciences \\ at West Virginia University \\ in partial fulfillment of the requirements \\ for the degree of
}

\author{
Master of Science \\ in \\ Psychology
}

\author{
Karen G. Anderson, Ph.D., Chair \\ Andy Lattal, Ph.D. \\ Hawley Montgomery-Downs, Ph.D. \\ Department of Psychology
}

Morgantown, West Virginia

2011

Keywords: choice; delay discounting; diazepam; Fischer 344; impulsive; Lewis; rat; self-control; temporal discounting 


\section{ABSTRACT \\ Effects of Acute and Repeated Administration of Diazepam on \\ Delay Discounting in Lewis and Fischer 344 Rats}

Sally L. Huskinson

Impulsive choice is often examined using a delay-discounting procedure where choice is between two reinforcers of different magnitudes presented at varying delays. Individual discounting rates can be influenced by many factors including strain differences and drug effects. Lewis (LEW) and Fischer 344 (F344) rats have behavioral and neurochemical differences relevant to delay discounting and were used to examine effects of acute and repeated diazepam administration on impulsive choice. Consistent with previous literature, largerreinforcer choice decreased as a function of increasing delays for all rats, and steeper discounting functions occurred for LEW relative to F344 rats. Acute and repeated diazepam administration resulted in differential effects between rat strains and sometimes between subjects within the same rat strain. Overall, larger-reinforcer choice was unchanged across multiple phases of the experiment for LEW rats, with some exceptions during the acute phase. For F344 rats, largerreinforcer choice increased following acute administration of smaller doses of diazepam and decreased following acute administration of the largest dose tested. Decreases in largerreinforcer choice occurred for F344 rats during repeated conditions and persisted throughout a non-drug baseline phase. Results from the present study have raised more questions about, and potential directions for future investigation of, multiple environmental, genetic, and neurochemical variables involved in delay discounting and effects of benzodiazepines on delay discounting. 


\section{Acknowledgements}

I would like to thank Karen Anderson, Andy Lattal, and Hawley Montgomery-Downs for serving as members of my thesis committee and for their valuable input and comments in preparing this manuscript. I am especially thankful to Karen for serving as the chair of my committee and as my advisor. 
Table of Contents

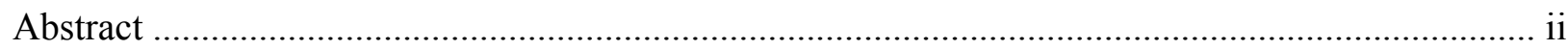

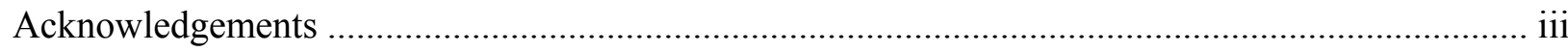

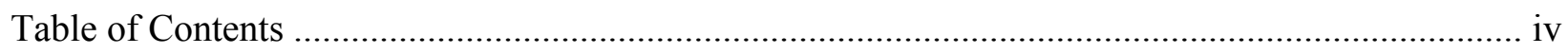

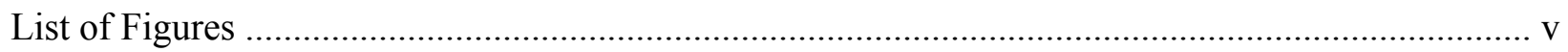

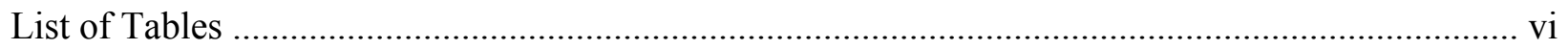

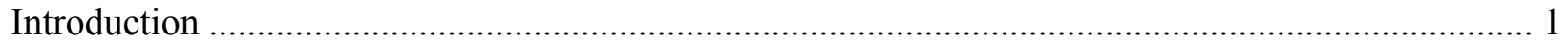

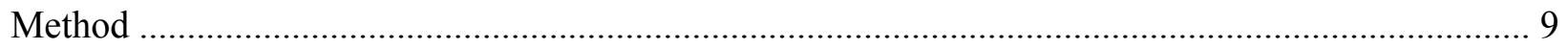

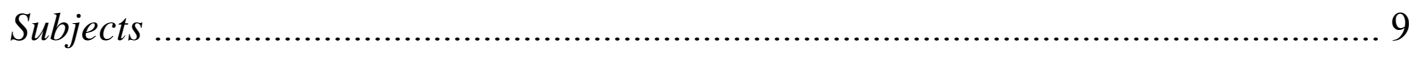

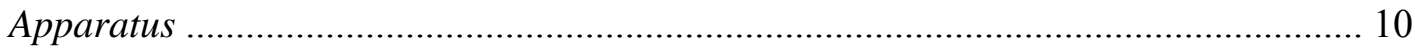

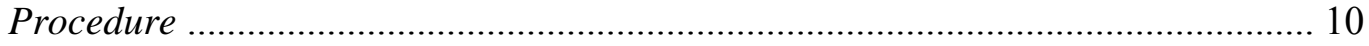

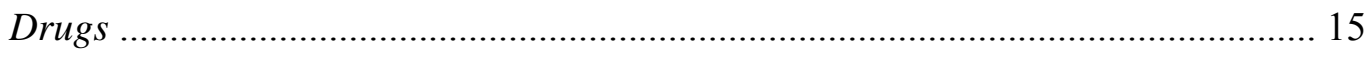

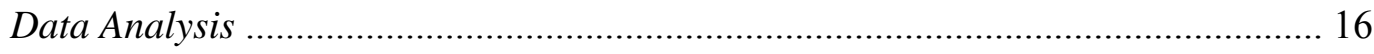

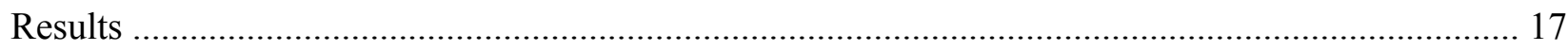

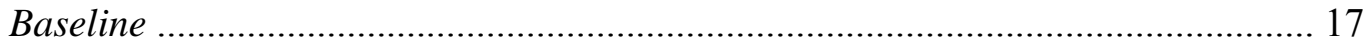

Determination of the Acute Dose-Response Function............................................. 21

Repeated (Chronic) Drug Administration........................................................ 28

Determination of the Chronic Dose-Response Function......................................... 31

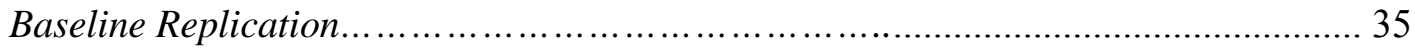

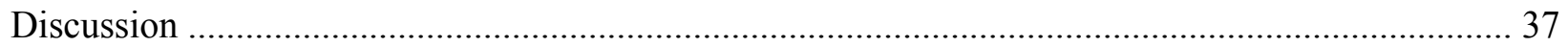

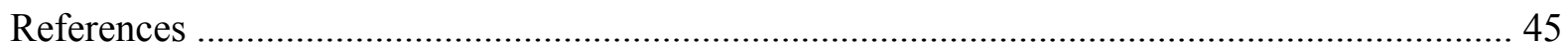


List of Figures

Figure 1. Mean percent larger-reinforcer choice as a function of delay blocks for the last five baseline sessions (circles) and for 0-sec probe sessions (squares) conducted during the terminal (baseline) delay series for LEW (closed symbols) and F344 (open symbols) rats. Error bars represent standard error of the mean. The x-axis is composed of blocks rather than delay values as two LEW and three F344 rats responded in a 0, 5, 10, 20, and 40-s delay series, and six LEW and five F344 rats responded in a $0,2,4,8$, and 16-s delay series. During 0 -s probe sessions the delay to the larger reinforcer was $0 \mathrm{~s}$ across all five blocks of trials

Figure 2. Mean indifference points (left panel) and AUC (right panel) from the last five sessions of the terminal (baseline) delay series for LEW (filled bars) and F344 (open bars) rats. Error bars represent standard error of the mean. Single and double asterisks represent strain differences at $p<.05$ and $p<.01$, respectively.

Figure 3. Mean percent larger-reinforcer choice as a function of delay for acute diazepam administration, including vehicle (closed squares), 1.0 (open circles), 3.0 (open triangles), and 10.0 (gray diamonds) $\mathrm{mg} / \mathrm{kg}$ of diazepam for LEW (left panel) and F344 (right panel) rats. Error bars represent standard error of the mean. The x-axis is composed of blocks rather than delay values as two LEW and three F344 rats responded in a 0, 5, 10, 20, and 40-s delay series, and six LEW and four F344

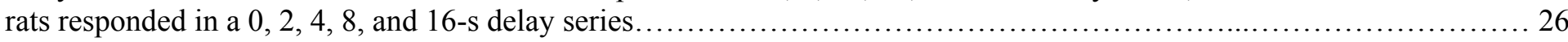

Figure 4. Mean indifference points (top panel) and AUC (bottom panel) as a function of doses of diazepam (control, vehicle, $1.0,3.0$, and $10.0 \mathrm{mg} / \mathrm{kg}$ ) during acute drug administration for LEW (closed symbols) and F344 (open symbols) rats. Error bars represent standard error of the mean. Single and double asterisks represent significance levels of $p<.05$ and $p<.01$, respectively, where differences occurred between rat strains at a particular dose. Single and double crosses represent significance levels of $p<.05$ and $p<.01$, respectively, where significant changes for a particular dose occurred relative to vehicle for each strain separately.

Figure 5. Mean percent larger-reinforcer choice as a function of delay during acute vehicle administration (closed squares) and the first (open triangles) and last (gray triangles) five sessions of repeated diazepam administration. Error bars represent standard error of the mean. The x-axis is composed of blocks as one LEW and three F344 rats responded in a 0, 5, 10, 20, and 40-s delay series, and six LEW and three F344 rats responded in a 0, 2, 4, 8, and 16-s delay

series

Figure 6. Mean percent larger-reinforcer choice as a function of delay during determination of the chronic dose-response function, including vehicle (closed squares), 1.0 (open circles), 3.0 (open triangles), and 10.0 (gray diamonds) $\mathrm{mg} / \mathrm{kg}$ of diazepam for LEW (left panel) and F344 (right panel) rats. Error bars represent standard error of the mean. The X-axis is composed of blocks rather than delay values as one LEW and three F344 rats responded in a 0, 5, 10, 20, and 40-s delay series, and six LEW and three F344 rats responded in a 0, 2, 4, 8, and 16-s delay series....

Figure 7. Mean indifference points (top panels) and AUC (bottom panels) as a function of doses of diazepam (vehicle, 1.0, 3.0 , and $10.0 \mathrm{mg} / \mathrm{kg}$ ) during acute (closed symbols) and chronic (open symbols) drug administration for LEW (left panels) and F344 (right panels) rats. Error bars represent standard error of the mean. Single and double asterisks represent significance levels of $p<.05$ and $p<.01$, respectively, where differences occurred between acute and chronic administration

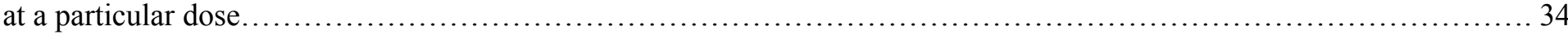

Figure 8. Mean percent larger-reinforcer choice as a function of delay blocks for the last five sessions conducted during the terminal (baseline) delay series (circles) and last five sessions from the baseline replication phase (squares) for only those LEW (left panel) and F344 (right panel) rats that completed all phases of the experiment. Error bars represent standard error of the mean. The x-axis is composed of blocks rather than delay values as one LEW and three F344 rats responded in a 0 , 5 , 10, 20, and 40-s delay series, and six LEW and three F344 rats responded in a 0, 2, 4, 8, and 16-s delay series............ 36 


\section{List of Tables}

Table 1. Subject, terminal delay series (Delay Series), number of 0-s probe sessions conducted during the terminal delay series ( 0 -s probes Baseline), number of sessions during the terminal delay series (Baseline), number of 0 -s probe sessions conducted during the acute phase ( 0 -s probes Acute), number of sessions during determination of the acute dose-response function (Acute DEC), the chronic dose selected for individual subjects (Chronic Dose), number of sessions during repeated diazepam administration for 30-50 sessions (Repeated), number of sessions during determination of the chronic dose-response function (Chronic DEC), number of 0 -s probe sessions conduction after completion of chronic diazepam administration ( 0 -s probes), and number of sessions conducted during the baseline replication at the end of experimentation (Baseline 2). Means and standard error of the means (SEM) are shown for each strain separately

Table 2. Subject, terminal delay series (Delay Series), and indifference points for each subject during the terminal delay series (Baseline), determination of the acute dose-response function (Control, VEH, 1.0, 3.0, and $10.0 \mathrm{mg} / \mathrm{kg}$ ), first five (First 5 Repeated) and last five (Last 5 Repeated) sessions of repeated administration of diazepam for 30-50 sessions, determination of the chronic dose-response function (VEH, 1.0,3.0, and $10.0 \mathrm{mg} / \mathrm{kg}$ ), and baseline replication at the end of experimentation (Baseline 2) ............................................. 24

Table 3. Subject, terminal delay series (Delay Series), and AUC for each subject during the terminal delay series (Baseline), determination of the acute dose-response function (Control, $\mathrm{VEH}, 1.0,3.0$, and $10.0 \mathrm{mg} / \mathrm{kg}$ ), first five (First 5 Repeated) and last five (Last 5 Repeated) sessions of repeated administration of diazepam for 30-50 sessions, determination of the chronic dose-response function ( $\mathrm{VEH}, 1.0,3.0$, and $10.0 \mathrm{mg} / \mathrm{kg}$ ), and baseline replication at the end of

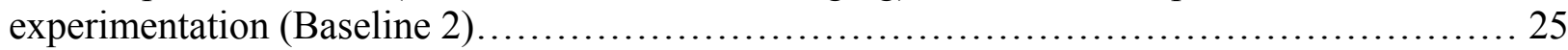




\section{Introduction}

Behavior identified as impulsive is associated with psychological and behavioral disorders such as attention-deficit/hyperactivity disorder (ADHD), gambling, substance abuse, eating disorders, and others (Evenden, 1999; Evenden \& Ryan, 1996; Kollins, 2003; Perry \& Carroll, 2008; Reynolds, 2006). A greater understanding of neurological, genetic, and behavioral mechanisms that underlie impulsive behavior may enhance the understanding and treatment of problem disorders. Impulsive behavior is correlated with substance abuse, though it is unclear whether high rates of impulsive behavior leads to substance abuse, substance abuse leads to high rates of impulsive behavior, or if both impulsive behavior and substance abuse are related through an unknown third variable. In the laboratory, a delay-discounting procedure is often used to assess impulsive choice in both human and animal models (e.g., Ainslie, 1975; Bickel, Odum, \& Madden, 1999; Mazur, 1987).

\section{Delay-Discounting Procedure}

Using the delay-discounting procedure, impulsive choice is examined by providing subjects with a choice between two reinforcers of different magnitudes presented at varying delays (Ainslie, 1975; Anderson \& Woolverton, 2005; Mazur, 1987). Impulsive choice in this context is operationally defined as choosing a smaller, more immediate reinforcer over a larger, more delayed reinforcer, and an indifference point, or the delay value at which the smaller and larger reinforcer are chosen equally, can be interpolated. The effectiveness of the larger delayed reinforcer is said to be devalued, or discounted, as a function of delay, and its subjective value may become less than the smaller immediate reinforcer. Shorter indifference points or steeper discounting curves indicate higher rates of impulsive choice.

Many argue that delay discounting is best described by the hyperbolic equation: $V=A /(1$ 
$+k D$ ), where $V$ represents reinforcer value, $A$ represents reinforcer amount, $D$ represents reinforcer delay, and $k$ is a free parameter used to indicate the rate of decrease in value, or rate of discounting (e.g., Mazur, 1987; Myerson \& Green, 1995; Perry \& Carroll, 2008). This equation predicts that the value of a reinforcer decreases faster at shorter delays and more slowly at longer delays as a function of delay to its presentation, and higher $k$ values indicate steeper discounting rates. Many studies, some of which are described below, have utilized this hyperbolic model for various comparisons of discounting rates between different subjects, different magnitudes of reinforcers, or qualitatively different reinforcers with both human and non-human animals.

\section{Drug Exposure and Delay Discounting}

Numerous studies find steeper delay-discounting functions, which indicate a more rapid devaluation of the larger reinforcer as a function of delay, in substance users compared to nonusers (see Reynolds, 2006 for a review). For example, Kollins (2003) examined choice between an adjusting amount of hypothetical money delivered immediately versus a larger amount delivered after a delay. Steeper discounting curves were associated with self-report measures of younger first use of cigarettes, alcohol, and marijuana, higher rates of "passing out" from alcohol use, and higher number of lifetime illicit drug use. Other studies targeting more specific drug classes indicate that alcohol (Petry, 2001), nicotine (Bickel et al., 1999; Mitchell, 1999; Reynolds, Richards, Horn, \& Karraker, 2004), opiate (Madden, Petry, Badger, \& Bickel, 1997; Madden, Bickel, \& Jacobs, 1999), cocaine (Coffey, Gudleski, Saladin, \& Brady, 2003), and methamphetamine abusers (Hoffman et al. 2006; Monterosso et al. 2007) discount delayed reinforcers more steeply than non-users, or ex-users (see Reynolds, 2006 for a review). Drugdependent participants also discounted hypothetical cigarettes (Bickel et al., 1999), heroin (Madden et al., 1997; Madden et al., 1999), and cocaine (Coffey et al., 2003) at a significantly 
higher rate than hypothetical money.

Laboratory research with non-human animals indicates a relation between drug selfadministration and delay discounting. Rats categorized as high on impulsive choice subsequently self-administered more freely available, orally delivered ethanol (Poulos, Le, \& Parker, 1995), more quickly acquired intravenous cocaine self-administration, and over the last five sessions of acquisition, self-administered more cocaine compared to rats categorized as low impulsive (Perry, Larson, German, Madden, \& Carroll, 2005; Perry, Nelson, \& Carroll, 2008). Additionally, rats categorized as high impulsive lever pressed more for nicotine infusions at fixed-ratio (FR) values of 20 and 25 compared to those categorized as low impulsive (Diergaarde et al., 2008). These results suggest that higher rates of impulsive choice may precede substance abuse. Other laboratory research has shown that exposure to a drug, via experimenter administration, may affect performance on subsequent delay-discounting tasks. Simon, Mendez, and Setlow (2007) examined two groups of rats exposed to either a high dose of cocaine (30 $\mathrm{mg} / \mathrm{kg}$ ) or saline for 14 days followed by three weeks with no testing or dosing. The group of rats exposed to cocaine chose the larger-reinforcer less often than the group exposed to saline. This suggests that exposure to a commonly abused drug like cocaine may somehow alter impulsive choice.

\section{Behavioral Differences Between Lewis and Fischer 344 rats}

Researchers have examined roles of behavioral and genetic factors on impulsive choice and their relation to substance use by comparing different rat strains. Comparisons between Lewis (LEW) and Fischer 344 (F344) rats provide evidence of behavioral differences that may be relevant to delay discounting and substance abuse. Using a delay-to-reinforcement procedure for acquisition of lever pressing, Anderson and Elcoro (2007) found that more LEW than F344 
rats acquired the response. Compared to F344 rats, LEW rats consume more freely available food adulterated with morphine and codeine than standard unadulterated rat chow (Suzuki, Otani, Koike, \& Misawa, 1988) and have higher progressive-ratio breakpoints for intravenous administration of morphine across various doses (Garcia-Lecumberri et al., 2010). LEW rats more readily acquire a lever press maintained by intravenous cocaine administration and initially self-administer larger amounts of cocaine than F344 rats (Freeman, Kearns, Kohut, \& Riley, 2009; Kosten et al., 1997). Once self-administration of cocaine is established, however, LEW rats self-administer less, have lower progressive-ratio breakpoints, and more elastic demand curves (Christensen, Kohut, Handler, Silberberg, \& Riley, 2009; Freeman et al. 2009; Kosten, Zhang, \& Haile, 2007). When ethanol was a consequence for lever pressing, LEW rats had higher response rates and oral intake of ethanol under various FR schedules than F344 rats (Suzuki, George, \& Meisch, 1988).

LEW rats also have shorter indifference points compared to F344 rats when utilizing a within-session adjusting-delay procedure where delay to the larger reinforcer systematically increased within single sessions (Anderson \& Diller, 2010; Anderson \& Woolverton, 2005; Garcia-Lecumberri et al., 2010; Huskinson, Krebs, \& Anderson, revisions submitted). This finding was replicated by systematically increasing delays between sessions (Madden, Smith, Brewer, Pinkston, \& Johnson, 2008). Using an adjusting-amount procedure, however, LEW and F344 rats did not differ in baseline levels of impulsive choice (Wilhelm \& Mitchell, 2009).

\section{Role of Dopamine and Serotonin in Delay Discounting}

LEW and F344 rats have neurochemical differences that may be relevant to delay discounting. LEW rats have fewer dopamine (DA) receptors (specifically $\mathrm{D}_{2}$ and $\mathrm{D}_{3}$ ) and $\mathrm{DA}$ transporters in some brain regions than F344 rats (Flores, Wood, Barbeau, Quiron, \& Srivastava, 
1998), and LEW rats have lower levels of serotonin (5-HT) and fewer $5-\mathrm{HT}_{1 \mathrm{~A}}$ receptors in some brain regions compared to F344 rats (Burnet, Mefford, Smith, Gold, \& Sternberg, 1992; Selim \& Bradberry, 1996). Previous research examining neurochemical factors that affect impulsive choice suggest that these systems play important roles in impulsive behaviors (see Cardinal, Robbins, \& Everitt, 2003 for a review). Depletion of DA in rats by injection of 6hydroxydopamine (6-OHDA) into the orbital prefrontal cortex (Kheramin et al., 2004) and depletion of 5-HT by injection of the neurotoxin 5,7-dihydroxytryptamine (5,7-DHT) in the dorsal and median raphe nuclei (Mobini et al., 2000; Wogar, Bradshaw, \& Szabadi, 1993) increased choice of a smaller, immediate reinforcer at shorter delays compared to rats that received sham lesions. Delay discounting was not affected by global 5-HT depletion when intracerebroventricular infusions of 5,7-DHT or vehicle were administered to rats matched according to baseline performance (Winstanley, Dalley, Theobald, \& Robbins, 2003). Global 5HT depletion did, however, inhibit the ability of $d$-amphetamine, an indirect DA agonist, to increase larger-reinforcer choice in the lesioned compared to non-lesioned animals (Winstanley et al., 2003).

To further support the role of DA and 5-HT in impulsive choice, there are indications that acute administration of DA and 5-HT drugs, particularly psychomotor stimulants such as $d$ amphetamine, may affect delay discounting, though results have been mixed. Increases in larger-reinforcer choice occurred following administration of $d$-amphetamine (Cardinal, Robbins, \& Everitt, 2000; Huskinson et al., revisions submitted; Wade, de Wit, \& Richards, 2000; Winstanley et al., 2003) and dexfenfluramine, a 5-HT releaser (Poulos, Parker, \& Le, 1996). However, administration of $d$-amphetamine and DOI, a 5-HT 2 agonist, also decreased larger-reinforcer choice (Cardinal et al., 2000; Evenden \& Ryan, 1996; 1999; Slezak \& 
Anderson, 2009). DA antagonists, flupenthixol and raclopride, decreased larger-reinforcer choice (Cardinal et al.; Wade et al., 2000), and SCH 23390, also a DA antagonist, had no effect (Wade et al.). It is possible that different baseline rates of delay discounting (Perry, Stairs, \& Bardo, 2008) and presence or absence of a cue during the delay (Cardinal et al.) may account for these discrepancies. The interaction between DA and 5-HT drugs and delay discounting requires additional research.

\section{CNS Depressants: Effects on Dopamine and Serotonin}

Compared to psychomotor stimulants, such as $d$-amphetamine, there is much less research regarding what effects administration of central nervous system (CNS) depressants such as ethanol and benzodiazepines would have on impulsive choice. Both ethanol and benzodiazepines have facilitative actions on the CNS inhibitory neurotransmitter system gammaAminobutyric acid (GABA), with specific action at the $\mathrm{GABA}_{\mathrm{A}}$ receptor site (e.g., Lobo \& Harris, 2008), in addition to actions on DA and 5-HT systems. Acute administration of ethanol increased extracellular levels of DA and 5-HT in the nucleus accumbens of Wistar rats (Yoshimoto, McBride, Lumeng, \& Li, 1992) and increased extracellular DA in the nucleus accumbens in F344 but not LEW rats (Mocsary \& Bradberry, 1996). The 5-HT agonist parachloroamphetamine can reduce impulsive behavior induced by ethanol (Olmstead, Hellemans, \& Paine, 2006), and ethanol self-administration increased levels of extracellular DA in Long-Evans and Wistar rats (Doyon, Anders, Ramachandra, Czachowski, \& Gonzales, 2005; Weiss, Lorang, Bloom, \& Koob, 1993).

Benzodiazepines decreased 5-HT neuronal activity and release in specific brain regions (Stein, Wise, \& Belluzzi, 1975). Administration of diazepam, a benzodiazepine, decreased basal levels of extracellular DA in the prefrontal cortex of rats (Finlay, Zigmond, \& Abercrombie, 
1995) and attenuated extracellular levels of DA and 3,4-dihydroxyphenylacetic acid (a DA metabolite) that were increased in the prefrontal cortex of rats via acute tail pressure and foot shock, respectively (Finlay et al., 1995; Roth, Tam, Ida, Yang \& Deutch, 1988). Benzodiazepines may also differentially affect LEW and F344 rats. LEW rats developed less physical dependence and withdrawal compared to F344 rats following repeated exposure and removal of food mixed with diazepam (Suzuki, Lu, Motegi, Yoshii, \& Misawa, 1992).

\section{CNS Depressants and Delay Discounting}

Results from acute administration of ethanol and benzodiazepines on delay discounting in humans are mixed. Using an adjusting-amount task, Richards, Zhang, Mitchell, and de Wit (1999) found no differences in impulsive choice following oral administration of different doses of alcohol or placebo. Conversely, Ortner, MacDonald, and Olmstead (2003) found that blood alcohol level was negatively correlated with discounting slope. Following consumption of 0.7 $\mathrm{g} / \mathrm{kg}$ of alcohol, a placebo drink, or no drink, participants in the alcohol group had higher indifference points compared to the non-alcohol groups. These results suggest that acute alcohol administration increases self-controlled choice in humans. Administration of therapeutically effective doses of diazepam ( 5 and $10 \mathrm{mg}$ ) and larger doses of diazepam $(20 \mathrm{mg})$ did not affect rates of delay discounting in humans (Reynolds, Richards, Dassinger, \& de Wit, 2004; Acheson, Reynolds, Richards, \& de Wit, 2006).

Effects of acute ethanol and benzodiazepine administration on impulsive choice in rats are also mixed. Using a T-maze procedure, Olmstead and colleagues (2006) examined effects of alcohol on choice between two food pellets delivered immediately, and twelve food pellets delivered after a 10-s delay. Alcohol, at all doses tested $(0.6-1.8 \mathrm{~g} / \mathrm{kg})$, decreased largerreinforcer choice relative to vehicle administration. Also using a T-maze procedure, Bizot, 
Bihan, Puech, Hamon, and Theibot (1999) found that bretazenil, a partial benzodiazepine agonist, reduced larger-reinforcer choice at a 15-s delay while alprazolam, a full benzodiazepine agonist, had no effects at a 15 -s delay and increased larger-reinforcer choice at a 25 -s delay. Diazepam and muscimol, a GABA agonist, decreased larger-reinforcer choice at a 15-s delay and had no effect at a 25-s delay (Bizot et al., 1999). Other researchers employing a T-maze procedure with rats found that diazepam decreased larger-reinforcer choice at 15 and 30-s delays, and other benzodiazepines (nitrazepam, chlordiazepoxide, and clobazam) have the same effect at a 15-s delay (Thiebot, Bihan, Soubrie, \& Simon, 1985).

Using a different delay-discounting procedure, where rats chose between a lever that delivered a smaller reinforcer immediately versus a lever that delivered a larger reinforcer after varying delays, ethanol decreased larger-reinforcer choice relative to saline (Tomie, Aguado, Pohorecky, \& Benjamin, 1998) and water (Evenden \& Ryan, 1999). Administration of chlordiazepoxide also decreased larger-reinforcer choice depending on dose and presence of a cue signaling the delay (Cardinal et al., 2000), while diazepam increased larger-reinforcer choice (Evenden \& Ryan, 1996) or had no effect (Charrier \& Thiebot, 1996). It is possible that the various procedures used resulted in the mixed findings across studies.

\section{Statement of the Problem}

Previous studies that examined substance abuse and its relation to delay discounting have included participants with a history of long-term drug exposure. It is possible that the increased impulsive choice observed in these participants developed as result of repeated exposure to drugs, although it cannot be ruled out that the participants had higher rates of impulsive choice prior to drug exposure. Using an animal model, it is possible to detect differences in impulsive choice during a baseline condition where animals have no previous drug exposure compared to impulsive choice following acute and repeated, experimenter-administered, drug exposure. 
Although numerous studies have examined effects of stimulant drugs on impulsive choice, relatively few have examined effects of acute and repeated administration of CNS depressants, particularly benzodiazepines, and what effects they have on impulsive choice. Benzodiazepines such as diazepam are widely used as anxiolytics, muscle relaxants, anticonvulsants, and are often abused at larger doses (Woods, Katz, \& Winger, 1987). To date, no researchers have examined effects of experimenter-administered benzodiazepines on delay discounting with LEW and F344 rats. Using two rat strains known to differ in baseline rates of delay discounting and their neurochemical response to CNS depressants may give insight into behavioral and biological determinants of impulsive choice. The present experiment evaluated effects of exposure to acute and repeated administration of diazepam on delay discounting in LEW and F344 rat strains. This study was designed to assess whether LEW and F344 rats differ in percent larger-reinforcer choices at baseline, and whether acute or repeated administration of diazepam differentially affects percent larger-reinforcer choice.

\section{Method}

\section{Subjects}

Eight experimentally naïve male LEW rats and eight experimentally naïve male F344 rats (Harlan Sprague-Dawley, Inc., Indianapolis, IN) served as subjects. Rats were approximately three months old at the start of experimentation, and consistent with previous findings, LEW rats $(M=298 \mathrm{~g}, S E M=4.0)$ weighed slightly more than F344 rats ( $M=283 \mathrm{~g}, S E M=3.6)$ (e.g., Christensen et al., 2009). Overall, this strain difference was observed throughout experimentation. Rats were housed individually with free access to water in their home cages. Temperature and humidity were maintained at constant levels, and a reverse 12-hour light-dark cycle was in place. All sessions were conducted during the dark phase of the light-dark cycle at 
approximately the same time each day. The subjects were fed approximately 10-12 $\mathrm{g}$ of food one half hour following each experimental session. This schedule resulted in about 21 hours of food restriction prior to the start of each session.

\section{Apparatus}

Experimental sessions were conducted in eight standard operant-conditioning chambers for rats, each enclosed in a melamine sound-attenuating cubicle (Med Associates, VT). Each chamber contained a working area of $30.5 \mathrm{~cm}$ by $24.5 \mathrm{~cm}$ by $21.0 \mathrm{~cm}$, a grid floor, and a $45-\mathrm{mg}$ pellet dispenser with a pellet receptacle that was centered between two retractable response levers. The levers were $11.5 \mathrm{~cm}$ apart from each other and required at least a force $0.25 \mathrm{~N}$ for a response to be recorded. The levers were $4.8 \mathrm{~cm}$ wide, protruded $1.9 \mathrm{~cm}$ into the chamber, and were elevated $8 \mathrm{~cm}$ from the grid floor. Two $28-\mathrm{V}$ stimulus lights, $2.5 \mathrm{~cm}$ in diameter, were 7 $\mathrm{cm}$ above each lever. Each chamber contained a $28-\mathrm{V}$ houselight on the wall opposite to the wall containing the operandum. A ventilation fan circulated air and served to mask extraneous noise. Equipment was interfaced to a computer, and experimental sessions and data collection were programmed and conducted with MedPC-IV software (Med Associates, VT).

\section{Procedure}

Initial training. During initial lever-press training, food was delivered according to a conjoint variable-time (VT) 1-min, fixed-ratio (FR) 1 schedule. During these initial training sessions, both levers were extended into the chamber, and one 45-mg food pellet was delivered every minute on average and following a press on either lever. Sessions lasted until 60 food pellets were delivered. If at least 40 of the 60 total food pellet deliveries were not being earned according to the FR 1 component after four sessions under the conjoint VT 1-min, FR 1 schedule, lever pressing was shaped using reinforcement of successive approximations. 
Following the hand-shaping procedure, lever pressing was reinforced according to an FR 1 schedule with both levers extended, and a press on either lever resulted in delivery of a food pellet. Once lever pressing was established by either method (free-operant acquisition or hand shaping), it was reinforced according to an FR 1 schedule where only one lever had a programmed consequence of food delivery. After five food pellets were earned for presses on a single lever, an extinction schedule for that lever commenced, and the FR 1 contingency was alternated to the second lever. This alternating procedure occurred within each session until 40 food pellets were earned. Daily sessions continued until the subjects reliably earned food on both levers.

General procedure. After training sessions were complete, delay-discounting sessions began. Sessions began with a 10-min blackout period, during which the chamber was completely dark before the trials began. Following the blackout period, each session consisted of five blocks of eight trials each. Trials consisted of forced- and free-choice trials that started every $100 \mathrm{~s}$. The first two trials in each block were forced-choice trials with one, randomly determined, lever extended into the chamber, and the cue light directly above it illuminated. A lever press (FR 1) on the extended lever resulted in the lever being retracted into the chamber, the cue light darkening, and either a single food pellet was delivered immediately or three food pellets were delivered after a delay. At the start of the second forced-choice trial, the other lever was extended into the chamber, the cue light directly above it was illuminated, and the other outcome was available dependent on a single lever press (FR 1).

After exposure to both outcomes during the forced-choice trials, the remaining six trials in each block were free-choice trials. During these trials, both cue lights were illuminated, both levers were extended into the chamber, and the subject was allowed to choose one alternative. 
The lever correlated with the larger reinforcer remained constant within and across sessions. A press on either lever (FR 1) resulted in both levers being retracted, both cue lights darkening, and one immediate or three delayed food pellets were delivered, depending on which lever was pressed. If no lever press occurred within $30 \mathrm{~s}$ of a trial onset, the trial was recorded as an omission, the levers were retracted, and the cue lights darkened.

After completion of the six free-choice trials within a block, the delays to the three-pellet option were increased across the session in consecutive blocks while the one-pellet option was always delivered immediately. In the first block of eight trials, the delay to the larger reinforcer was always $0 \mathrm{~s}$. All sessions started with a delay series of $0 \mathrm{~s}$ in all five blocks. When choice of the larger reinforcer was at least $80 \%$ in each block, and for no more than three sessions, the delays were increased to a series of $0,1,2,4$, and 6 s across blocks. The 6-s delay series remained in effect until the total number of larger-reinforcer choices during the free-choice trials in the 0 -s block was at or above $80 \%$. The delays were then increased to $0,2,4,8$, and $16 \mathrm{~s}$ across blocks.

After the 16-s delay series, the delay series was increased or decreased as necessary for individual subjects to obtain discounting functions with no ceiling or floor effects (i.e., near exclusive choice for the larger or smaller reinforcer). Only if ceiling effects were obtained during the 16-s delay series, were the delays increased to $0,5,10,20$, and $40 \mathrm{~s}$ across blocks, and if necessary, to $0,10,20,40$, and $60 \mathrm{~s}$ across blocks. Sessions ended after 40 total (10 forcedand 30 free-choice) trials and were initially conducted five days per week. Six months into the experiment, sessions were conducted seven days per week. No systematic differences were observed between those subjects that received five compared to seven sessions per week during the baseline phase. 
Baseline. Once choice was maintained at a single terminal delay series, a baseline was established. The terminal (baseline) delay series was in effect for a minimum of 20 sessions plus the number of sessions until responding was stable. Stability was defined as $80 \%$ or greater choice for the larger reinforcer in the 0 -s delay block, less than $20 \%$ variation in total largerreinforcer choice during each of the last five sessions from the grand mean, and no increasing or decreasing trends between the total number of larger-reinforcer choices for each session across the last five sessions. To ensure sensitivity to the variations in reinforcer magnitude, all delays during a single session were set equal to zero at least once per week ( 0 -s probe sessions) during exposure to the terminal delay series (Cardinal et al., 2000; Diller, Saunders, \& Anderson, 2008; Evenden \& Ryan, 1996; 1999). Levers associated with the larger-reinforcer delivery were counterbalanced across subjects such that half of each group had the left lever associated with the larger reinforcer and half of each group had the right lever associated with the larger reinforcer.

Determination of the acute dose-response function. After establishing stable baseline data, acute effects of diazepam administration were assessed. Either vehicle or diazepam (1.0, 3.0 , and $10.0 \mathrm{mg} / \mathrm{kg}$ ) were administered by intraperitoneal injection two times per week, separated by at least two sessions, as long as two criteria were met. First, at least $80 \%$ of lever presses occurred on the lever associated with the larger reinforcer in each block during sessions when all delays were set equal to zero. Second, during control sessions, the session immediately preceding vehicle or drug sessions, at least $80 \%$ of lever presses occurred on the lever associated with the larger reinforcer in the first block when both delays were $0 \mathrm{~s}$. Before the first diazepam administration, vehicle was administered at least twice. Initially, half of LEW and half of F344 rats received smaller doses of diazepam $(0.3,0.56$ and $1.0 \mathrm{mg} / \mathrm{kg})$ in descending order, and the 
other half received them in ascending order. Because these doses did not result in any observed behavioral effects, it became necessary to increase the doses to those listed above, and all analyses include only $1.0,3.0$, and $10.0 \mathrm{mg} / \mathrm{kg}$ of diazepam. Each of the latter doses of diazepam were determined at least twice for each subject, and intermediate doses (1.7 and 5.6 $\mathrm{mg} / \mathrm{kg}$ ) were administered when intermediate drug effects needed to be determined. A third or fourth determination of a particular dose occurred when there was a substantial amount of variability in larger-reinforcer choice between the first and second determinations.

Repeated (chronic) drug administration. Once acute drug effects were determined, a dose of diazepam that resulted in the largest change in percent larger-reinforcer choice and maintained responding at $80 \%$ or greater for the larger reinforcer in the first block was administered repeatedly (chronic dose). If no change in percent larger-reinforcer choice occurred during acute diazepam administration, the largest dose of diazepam that did not completely suppress responding was used. To determine effects of repeated exposure to diazepam, experimental sessions were conducted seven days per week, and the chronic dose was administered daily prior to the start of each session. Repeated administration occurred for a minimum of 30 sessions and until choice was stable or a maximum of 50 sessions to ensure approximately the same amount of repeated drug exposure across subjects. In this condition, stability was defined as no more than $20 \%$ variation in the total number of larger-reinforcer choices during each of the last five sessions from the grand mean and no increasing or decreasing trends in the total number of larger-reinforcer choices across the last five sessions. During this phase, the delays were no longer set equal to zero once per week.

Determination of the chronic dose-response function. Following repeated administration of the (single) chronic dose of diazepam, the dose-response function was 
redetermined to allow for comparisons of larger-reinforcer choice before and after repeated diazepam exposure. Two times per week, with at least two sessions in between, the repeated dose was substituted with one of the previously administered doses of diazepam $(1.0,3.0, \& 10.0$ $\mathrm{mg} / \mathrm{kg}$ ) and vehicle. Each dose and vehicle administration was determined at least twice for each subject. A third or fourth determination of a particular dose occurred if there was a substantial amount of variability in larger-reinforcer choice between the first and second determinations.

Baseline replication. At the end of drug experimentation, diazepam administration ceased, and subjects that completed the repeated phase were exposed to 0 -s probe sessions where all delays were set equal to $0 \mathrm{~s}$ across the five blocks of trials. These sessions were conducted until larger-reinforcer choice was at least $80 \%$ across all blocks for each subject. Once this criterion was met, conditions were the same as those during the terminal baseline phase. Sessions were conducted seven days per week, with the terminal delay series in effect, except once per week (Wednesdays) when 0-s probe sessions were conducted until larger-reinforcer choice was at least $80 \%$ across all blocks of trials. This phase was in effect for a minimum of 20 sessions with the exception of LEW-2 and LEW-8 that received 19 and 15 sessions, respectively, and until choice was stable. The stability criteria were identical to those used during the terminal baseline phase. LEW-2 and LEW-8 did not meet the minimum session requirement as a result of equipment malfunction, however, the choice data from the last five sessions met stability criteria.

\section{Drugs}

Diazepam was obtained from Sigma-Aldrich (St. Louis, MO, USA) and was dissolved in $0.9 \%$ sodium chloride plus varying concentrations of Tween $80(2,3,5$, and $10 \%)$. Diazepam was injected in a volume of $1.0 \mathrm{mg} / \mathrm{kg}$. Vehicle $(0.9 \%$ sodium chloride plus varying concentrations of Tween 80$)$ or diazepam $(1.0,3.0$ and $10.0 \mathrm{mg} / \mathrm{kg})$ was administered via 
intraperitoneal injections immediately prior to experimental sessions. For the largest dose of diazepam, three drops of ethanol were added to every $5.0 \mathrm{mls}$ of solution.

\section{Data Analysis}

Percent choice for the larger reinforcer was the main dependent measure used. From this measure, indifference points and area under the curve (AUC) were calculated for each subject in each condition. Delay-discounting functions for each condition were plotted as percent choice for the larger reinforcer across increasing delays in each block. Using nonlinear regression, a function was fit to the data, and indifference points (delay value at which choice for the larger and smaller reinforcer are equal) were interpolated based on these functions. AUC was derived by calculating the area of trapezoids that were formed by drawing vertical lines from the normalized $\mathrm{x}$-axis to each obtained percent choice for the larger reinforcer at each delay. The areas of these trapezoids were added together and divided by the entire possible area of the graph (Myerson, Green, \& Warusawitharana, 2001). It is important to note that because the x-axis was normalized when calculating AUC, this measure does not take into account different delay series used in the present study. By using this measure, all delay series were transformed into a scale that ranged from zero to one.

To assess strain differences at baseline and following acute and repeated administration of diazepam on delay discounting, one-way analysis of variance (ANOVA) were conducted. To assess drug affects within each strain separately, repeated measures ANOVAs were conducted. Rat strain was a between-subjects variable and indifference points and AUC for acute and repeated doses of diazepam were within-subjects variables. Planned comparisons were made between each dose of diazepam and vehicle and between each repeated dose compared to each acute dose. Independent samples t-tests were conducted to examine differences in the number of 
0-s probe sessions and number of sessions in each condition between LEW and F344 rats.

\section{Results}

\section{Baseline}

The number of sessions conducted during the terminal baseline condition and number of 0 -s probe sessions conducted during baseline for individual subjects are presented in Table 1 . There was no difference between the number of sessions for LEW and F344 rats to reach stability during the terminal baseline condition. LEW rats did, however, require more 0 -s probe sessions during the terminal baseline condition compared to F344 rats $[t(14)=3.10, p<.01]$.

Figure 1 shows mean percent larger-reinforcer choice as a function of delay to the larger reinforcer (displayed as blocks of trials) for LEW (closed symbols) and F344 (open symbols) rats during the last five sessions of the terminal baseline (circles) and for all 0 -s probe sessions (squares) conducted during the terminal baseline condition. During 0 -s probe sessions, mean larger-reinforcer choice remained above $80 \%$ across all blocks of trials for both rat strains. During sessions when the delay to the larger reinforcer increased, choice for that alternative decreased for all subjects, and this occurred to a greater extent for LEW compared to F344 rats. The finding that more impulsive choices occurred for LEW compared to F344 rats is also indicated in Figure 2 which shows indifference points (left panel) and AUC (right panel) for each rat strain during the terminal baseline condition (see Tables 2 and 3 for indifference points and AUC, respectively, for individual subjects). LEW rats had shorter indifference points ( $M=6.6 \mathrm{~s}$, $S E M=0.8 \mathrm{~s})$ and smaller AUCs $(M=0.40, S E M=0.04)$ compared to $\mathrm{F} 344$ rats $(M=17.3 \mathrm{~s}$, $S E M=4.4 \mathrm{~s} ; M=0.61, S E M=0.04$, respectively) $[F(1,15)=5.88, p<.05 ; F(1,15)=16.34, p<$ .01 , respectively]. 


\section{Table 1}

Subject, terminal delay series (Delay Series), number of 0-s probe sessions conducted during the terminal delay series (0-s probes Baseline), number of sessions during the terminal delay series (Baseline), number of 0-s probe sessions conducted during the acute phase (0-s probes Acute), number of sessions during determination of the acute dose-response function (Acute DEC), the chronic dose selected for individual subjects (Chronic Dose), number of sessions during repeated diazepam administration for 30-50 sessions (Repeated), number of sessions during determination of the chronic dose-response function (Chronic DEC), number of 0-s probe sessions conduction after completion of chronic diazepam administration (0-s probes), and number of sessions conducted during the baseline replication at the end of experimentation (Baseline 2). Means and standard error of the means (SEM) are shown for each strain separately

\begin{tabular}{|c|c|c|c|c|c|c|c|c|c|c|}
\hline Subject & $\begin{array}{l}\text { Delay } \\
\text { Series }\end{array}$ & $\begin{array}{l}0 \text {-s probes } \\
\text { Baseline }\end{array}$ & Baseline & $\begin{array}{l}0 \text {-s probes } \\
\text { Acute }\end{array}$ & $\begin{array}{l}\text { Acute } \\
\text { DEC }\end{array}$ & $\begin{array}{c}\text { Chronic Dose } \\
(\mathrm{mg} / \mathrm{kg})\end{array}$ & Repeated & $\begin{array}{l}\text { Chronic } \\
\text { DEC }\end{array}$ & $\begin{array}{c}0-\mathrm{s} \\
\text { probes }\end{array}$ & Baseline 2 \\
\hline LEW-1 & 16 & 15 & 20 & 24 & 82 & 1.7 & 32 & 39 & 1 & 22 \\
\hline LEW-2 & 40 & 16 & 53 & 14 & 57 & 10.0 & 43 & 20 & 1 & 19 \\
\hline LEW-3 & 16 & 33 & 35 & 30 & 61 & 10.0 & 32 & 22 & 2 & 22 \\
\hline LEW-4 & 16 & 4 & 22 & 5 & 30 & 10.0 & 31 & 25 & 1 & 21 \\
\hline LEW-5 & 16 & 27 & 34 & 29 & 68 & 10.0 & 32 & 39 & 3 & 20 \\
\hline LEW-6 & 16 & 16 & 29 & 36 & 71 & 5.6 & 36 & 46 & 2 & 29 \\
\hline LEW-7 & 40 & 15 & 38 & 16 & 57 & $--/--$ & $--/--$ & $--/--$ & $--/--$ & $--/--$ \\
\hline LEW-8 & 16 & 13 & 44 & 16 & 40 & 3.0 & 34 & 40 & 6 & 15 \\
\hline $\begin{array}{c}\text { LEW } \\
M(S E M)\end{array}$ & & $17.4(3.1)$ & $34.4(3.9)$ & $21.3(3.6)$ & $58.3(5.9)$ & & $34.3(1.6)$ & $33.0(3.5)$ & $2.3(0.7)$ & $21.1(4.2)$ \\
\hline F344-1 & 16 & 9 & 35 & 15 & 54 & 1.0 & 30 & 37 & 1 & 20 \\
\hline F344-2 & 16 & 9 & 29 & 16 & 46 & 1.0 & 33 & 41 & 3 & 23 \\
\hline F344-3 & 40 & 7 & 35 & 21 & 68 & 10.0 & 40 & 22 & 3 & 24 \\
\hline F344-4 & 40 & 5 & 23 & 21 & 63 & 10.0 & 35 & 29 & 2 & 24 \\
\hline F344-5 & 16 & 7 & 27 & 17 & 63 & 10.0 & 36 & 44 & 1 & 20 \\
\hline F344-6 & 16 & 6 & 37 & 6 & 73 & $--/--$ & $--/--$ & --/-- & $--/--$ & --/-- \\
\hline F344-7 & 40 & 10 & 21 & 15 & 51 & 5.6 & 30 & 31 & 2 & 20 \\
\hline F344-8 & 16 & 7 & 27 & $--/--$ & $--/--$ & $---/-$ & $--/--$ & $--/--$ & $--/--$ & $--/--$ \\
\hline $\begin{array}{c}\text { F344 } \\
M(S E M)\end{array}$ & & $7.5(0.6)$ & $29.3(2.1)$ & 15.9 (1.9) & $59.7(3.7)$ & & $34.0(1.6)$ & $34.0(3.3)$ & $2.0(0.4)$ & $21.8(0.8)$ \\
\hline
\end{tabular}




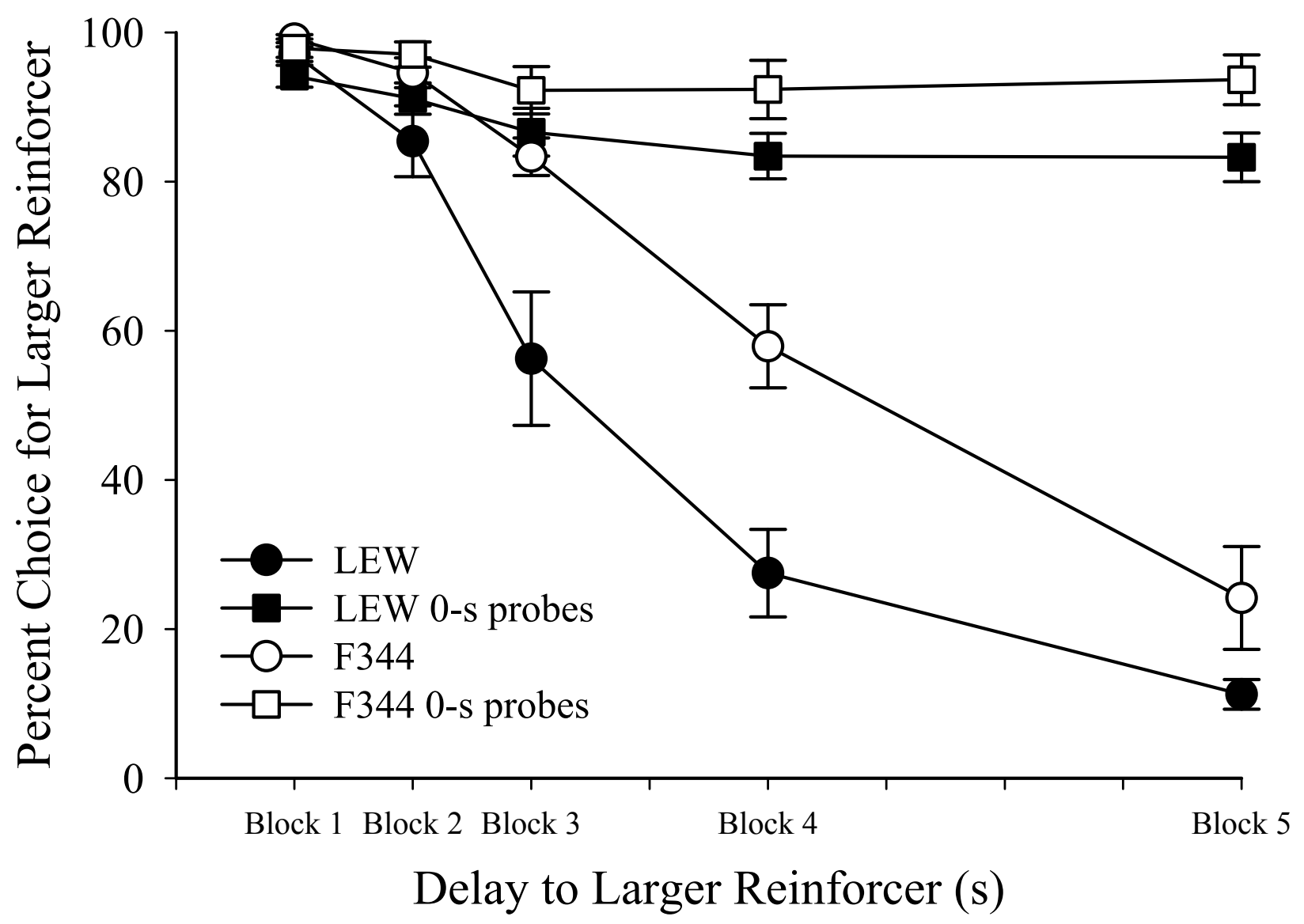

Figure 1. Mean percent larger-reinforcer choice as a function of delay blocks for the last five baseline sessions (circles) and for 0 -sec probe sessions (squares) conducted during the terminal (baseline) delay series for LEW (closed symbols) and F344 (open symbols) rats. Error bars represent standard error of the mean. The X-axis is composed of blocks rather than delay values as two LEW and three F344 rats responded in a 0, 5, 10, 20, and 40-s delay series, and six LEW and five F344 rats responded in a 0,2, 4, 8, and 16-s delay series. During 0-s probe sessions the delay to the larger reinforcer was $0 \mathrm{~s}$ across all five blocks of trials. 


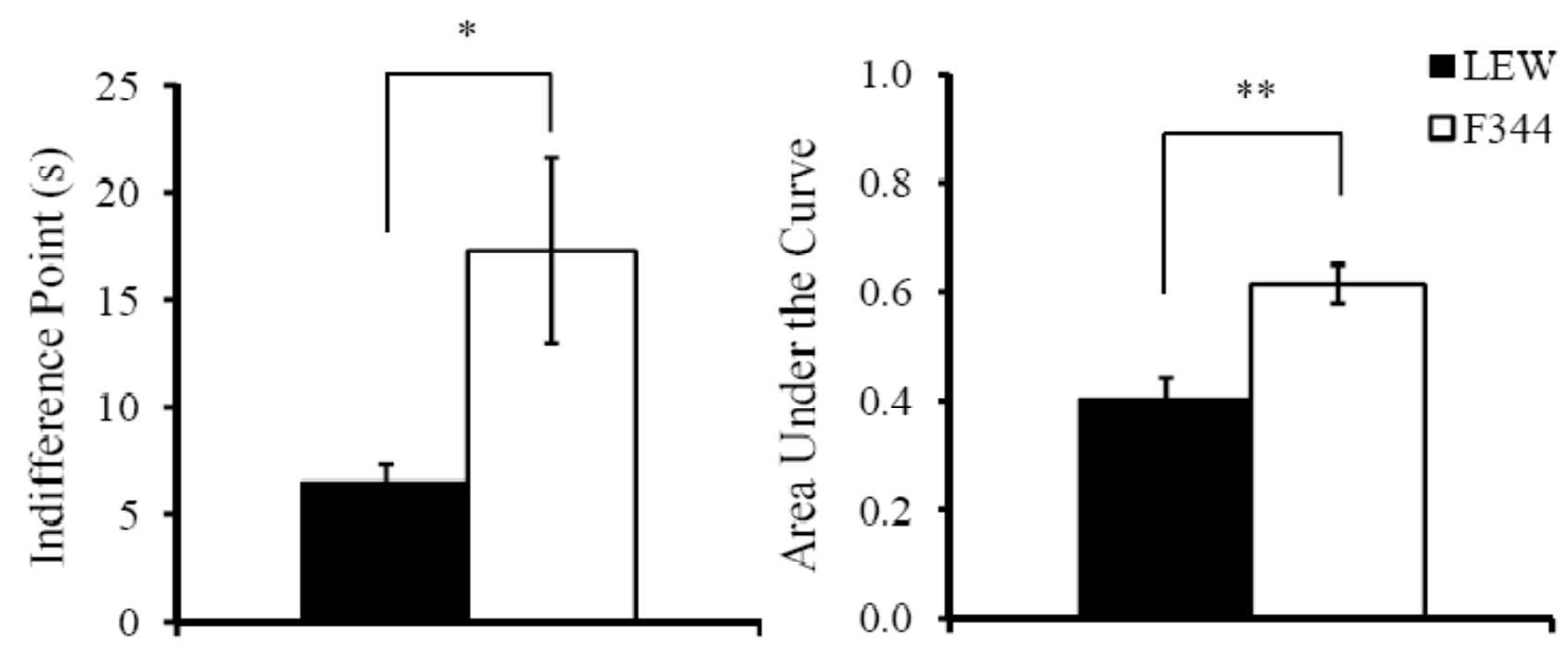

\section{Baseline}

Figure 2. Mean indifference points (left panel) and AUC (right panel) from the last five sessions of the terminal (baseline) delay series for LEW (filled bars) and F344 (open bars) rats. Error bars represent standard error of the mean. Single and double asterisks represent strain differences at $p<.05$ and $p<.01$, respectively 


\section{Determination of the Acute Dose-Response Function}

One F344 rat died before acute drug administration; acute analyses are therefore based on eight LEW and seven F344 rats. Table 1 shows the number of sessions conducted during the acute phase and number of 0 -s probe sessions conducted during this condition for individual subjects. There were no differences between the number of sessions for LEW and F344 rats to complete the acute drug administration phase or the number of 0 -s probe sessions conducted during this phase.

Figure 3 shows mean percent larger-reinforcer choice for vehicle and each dose of acute diazepam as a function of delay to the larger reinforcer (displayed as blocks of trials) for LEW (left panel) and F344 (right panel) rats during acute drug administration. For LEW rats, mean percent larger-reinforcer choice did not change relative to vehicle for any dose of diazepam administered, though it appears to trend toward an increase in larger-reinforcer choice following administration of the largest dose of diazepam tested $(10.0 \mathrm{mg} / \mathrm{kg})$. This trend is largely a result of choice for two subjects and is discussed further when results for indifference points and AUC are reported. For F344 rats, mean percent larger-reinforcer choice decreased (i.e., more impulsive choices) following acute administration of the largest dose of diazepam tested compared to vehicle administration.

The above findings are also indicated in Figure 4, which shows indifference points (top panel) and AUC (bottom panel) for LEW (closed symbols) and F344 (open symbols) rats as a function of acute doses of diazepam (see Tables 2 and 3 for individual-subject data). It is important to note that baseline strain differences in impulsive choice persisted throughout the acute drug phase as indifference points were shorter and AUC were smaller for LEW compared to $\mathrm{F} 344$ rats during control sessions (non-drug sessions) $[F(1,14)=7.88, p<.05 ; F(1,14)=6.75$, 
$p<.05$, respectively]. LEW rats also had shorter indifference points following vehicle administration relative to $\mathrm{F} 344$ rats $[F(1,14)=4.68, p=.05]$. Acute administration of diazepam had differential effects for each rat strain. For LEW rats, there was no significant effect of dose on indifference points or AUC. Following administration of the $10.0 \mathrm{mg} / \mathrm{kg}$ dose of diazepam, there was an increase in mean indifference points relative to vehicle administration, however, effects at this dose were not systematic across subjects. The mean was largely affected by two outliers; large increases in indifference points and AUC were observed for LEW-2 and LEW-3 at this dose relative to vehicle administration (see Tables 2 and 3 ).

For LEW rats, further examination of individual-subject data (see Tables 2 and 3), revealed that diazepam did affect larger-reinforcer choice, however, the effect occurred at different doses. For all subjects ( 8 of 8 ), at least one of the three doses administered increased AUC relative to vehicle. Statistical analysis comparing acute vehicle administration to the largest AUC obtained for each subject across the three doses administered revealed a significant increase in AUC $[F(1,7)=6.91, p<.05]$. The analysis included AUC at the $1.0 \mathrm{mg} / \mathrm{kg}$ dose for LEW-6 and LEW-7, at the $3.0 \mathrm{mg} / \mathrm{kg}$ dose for LEW-1, LEW-5, and LEW-8, and at the 10.0 $\mathrm{mg} / \mathrm{kg}$ dose for LEW-2, LEW-3, and LEW-4 relative to vehicle administration (see Table 3).

For F344 rats, diazepam had dose-dependent effects on indifference points and AUC $[F(4,24)=5.89, p<.01 ; F(4,24)=12.25, p<.01$, respectively $]$. Although not statistically significant, compared to vehicle administration, there was at least a slight increase in indifference points and AUC in six of seven and five of seven F344 rats following acute administration of 1.0 and $3.0 \mathrm{mg} / \mathrm{kg}$ of diazepam, respectively (see Tables 2 and 3). Indifference points and AUC did not increase following at least one of these doses for only one subject (F344-6). When this subject was not included in the analyses, AUC following $1.0 \mathrm{mg} / \mathrm{kg}$ were 
significantly greater than AUC following vehicle administration $[F(1,5)=7.66, p<.05]$, and indifference points following $3.0 \mathrm{mg} / \mathrm{kg}$ were significantly longer than indifference points following vehicle administration $[F(1,5)=6.84, p<.05]$. For $F 344$ rats, there was a significant decrease in indifference points and AUC following administration of the largest dose of diazepam $(10.0 \mathrm{mg} / \mathrm{kg})$ relative to vehicle administration $[F(1,6)=9.45, p<.05 ; F(1,6)=15.81$, $p<.01$, respectively]. The observed decrease in larger-reinforcer choice at the $10.0 \mathrm{mg} / \mathrm{kg}$ dose of diazepam relative to vehicle occurred for six of the seven F344 rats (see Tables 2 and 3). Comparisons between rat strains at each dose revealed that indifference points were shorter and AUC were smaller for LEW rats compared to F344 rats following administration of the $1.0 \mathrm{mg} / \mathrm{kg}$ dose of diazepam $[F(1,14)=6.71, p<.05 ; F(1,14)=10.31, p<.01$, respectively $]$. The opposite occurred at the largest dose of diazepam (i.e., greater impulsive choice for F344 rats). AUC at this dose decreased to a smaller value for $\mathrm{F} 344$ relative to $\operatorname{LEW}$ rats $[F(1,14)=$ 8.54, $p<.05]$. 
Table 2

Subject, terminal delay series (Delay Series), and indifference points for each subject during the terminal delay series (Baseline), determination of the acute dose-response function (Control, VEH, 1.0, 3.0, and $10.0 \mathrm{mg} / \mathrm{kg}$ ), first five (First 5 Repeated) and last five (Last 5 Repeated) sessions of repeated administration of diazepam for 30-50 sessions, determination of the chronic doseresponse function (VEH, 1.0, 3.0, and $10.0 \mathrm{mg} / \mathrm{kg}$ ), and baseline replication at the end of experimentation (Baseline 2).

\begin{tabular}{|c|c|c|c|c|c|c|c|c|}
\hline \multirow[b]{2}{*}{ Subject } & \multirow[b]{2}{*}{$\begin{array}{l}\text { Delay } \\
\text { Series } \\
\end{array}$} & \multicolumn{4}{|c|}{ Acute Dose (mg/kg) } & \multirow[b]{2}{*}{1.0} & \multirow[b]{2}{*}{3.0} & \multirow[b]{2}{*}{10.0} \\
\hline & & \multicolumn{2}{|c|}{ Baseline } & Control & VEH & & & \\
\hline LEW-1 & 16 & \multicolumn{2}{|c|}{7.67} & 9.78 & 7.89 & 9.04 & 10.78 & 9.57 \\
\hline LEW-2 & 40 & \multicolumn{2}{|c|}{10.25} & 10.17 & 10.92 & 9.73 & 12.85 & 63.20 \\
\hline LEW-3 & 16 & \multicolumn{2}{|c|}{8.09} & 6.84 & 5.89 & 8.34 & 14.52 & 20.66 \\
\hline LEW-4 & 16 & \multicolumn{2}{|c|}{4.27} & 5.05 & 5.30 & 5.25 & 5.25 & 6.76 \\
\hline LEW-5 & 16 & \multicolumn{2}{|c|}{3.52} & 4.01 & 4.92 & 4.90 & 5.40 & 3.19 \\
\hline LEW-6 & 16 & \multicolumn{2}{|c|}{7.38} & 5.33 & 6.18 & 6.70 & 3.11 & 2.65 \\
\hline LEW-7 & 40 & \multicolumn{2}{|c|}{6.33} & 8.89 & 9.35 & 8.68 & 7.97 & 9.46 \\
\hline LEW-8 & 16 & \multicolumn{2}{|c|}{4.91} & 6.07 & 3.73 & 4.75 & 7.41 & 5.00 \\
\hline$M(S E M)$ & & \multicolumn{2}{|c|}{$6.55(0.8)$} & $7.02(0.8)$ & $6.77(0.9)$ & $7.17(0.7)$ & $8.41(1.4)$ & $15.06(7.2)$ \\
\hline F344-1 & 16 & \multicolumn{2}{|c|}{8.88} & 7.34 & 4.96 & 7.59 & 5.26 & 5.26 \\
\hline F344-2 & 16 & \multicolumn{2}{|c|}{9.67} & 9.70 & 5.79 & 14.64 & 12.09 & 2.25 \\
\hline F344-3 & 40 & \multicolumn{2}{|c|}{42.88} & 12.98 & 15.00 & 17.05 & 18.61 & 5.35 \\
\hline F344-4 & 40 & \multicolumn{2}{|c|}{16.96} & 14.23 & 12.60 & 13.03 & 17.36 & 5.08 \\
\hline F344-5 & 16 & \multicolumn{2}{|c|}{8.26} & 7.45 & 6.51 & 8.61 & 5.56 & 3.00 \\
\hline F344-6 & 16 & \multicolumn{2}{|c|}{15.32} & 14.91 & 21.28 & 14.37 & 3.80 & 3.00 \\
\hline F344-7 & 40 & \multicolumn{2}{|c|}{27.95} & 21.91 & 21.04 & 35.20 & 28.90 & 1.53 \\
\hline F344-8 & 16 & \multicolumn{2}{|c|}{8.38} & $--/--$ & $--/--$ & $--/--$ & $--/--$ & --/-- \\
\hline \multicolumn{2}{|l|}{$M(S E M)$} & 17.29 & 4.4) & $12.65(1.9)$ & $12.45(2.6)$ & $15.78(3.5)$ & $13.08(3.5)$ & $3.64(0.6)$ \\
\hline & & & & Chronic Dos & $\mathrm{mg} / \mathrm{kg}$ ) & & & \\
\hline Subject & $\begin{array}{l}\text { Delay } \\
\text { Series }\end{array}$ & $\begin{array}{c}\text { First } 5 \\
\text { Repeated }\end{array}$ & $\begin{array}{c}\text { Last } 5 \\
\text { Repeated }\end{array}$ & VEH & 1.0 & 3.0 & 10.0 & Baseline 2 \\
\hline LEW-1 & 16 & 18.32 & 11.95 & 7.79 & 13.39 & 14.86 & 17.45 & 7.22 \\
\hline LEW-2 & 40 & 45.89 & 92.16 & 16.02 & 8.76 & 10.00 & 17.22 & 7.59 \\
\hline LEW-3 & 16 & 9.98 & 2.48 & 1.50 & 3.30 & 2.89 & 3.96 & 5.60 \\
\hline LEW-4 & 16 & 3.89 & 7.26 & 4.22 & 3.85 & 4.00 & 5.43 & 6.02 \\
\hline LEW-5 & 16 & 3.46 & 5.02 & 3.22 & 3.89 & 5.69 & 3.13 & 5.12 \\
\hline LEW-6 & 16 & 2.79 & 3.72 & 5.78 & 3.29 & 5.84 & 4.89 & 5.31 \\
\hline LEW-7 & 40 & $--/--$ & $--/--$ & $--/--$ & $--/--$ & $--/--$ & $--/--$ & $--/--$ \\
\hline LEW-8 & 16 & 5.22 & 6.04 & 4.50 & 5.60 & 6.51 & 4.93 & 4.96 \\
\hline$M(S E M)$ & & $12.79(5.9)$ & $18.38(12.4)$ & $6.15(1.8)$ & $6.01(1.4)$ & $7.11(1.5)$ & $8.14(2.4)$ & $5.97(0.4)$ \\
\hline F344-1 & 16 & 5.58 & 3.92 & 5.05 & 5.11 & 5.61 & 1.30 & 10.34 \\
\hline F344-2 & 16 & 9.89 & 5.44 & 3.24 & 4.54 & 5.28 & 2.62 & 4.14 \\
\hline F344-3 & 40 & 3.06 & 3.70 & 4.87 & 4.62 & 4.62 & 3.88 & 4.44 \\
\hline F344-4 & 40 & 0.29 & 2.27 & 6.80 & 5.35 & 2.99 & 1.39 & 7.13 \\
\hline F344-5 & 16 & 2.00 & 6.43 & 3.72 & 3.52 & 4.63 & 5.14 & 4.07 \\
\hline F344-6 & 16 & $--/--$ & $--/--$ & $--/--$ & $--/--$ & $--/--$ & $--/--$ & $--/--$ \\
\hline F344-7 & 40 & 5.37 & 4.00 & 4.82 & 6.12 & 3.43 & 3.43 & 5.43 \\
\hline F344-8 & 16 & --/-- & $--/--$ & $--/--$ & $--/--$ & $--/--$ & $--/--$ & $--/--$ \\
\hline$M(S E M)$ & & $4.37(1.8)$ & $4.29(0.6)$ & $4.75(0.5)$ & $4.88(0.4)$ & $4.43(0.4)$ & $2.96(0.6)$ & $5.93(1.0)$ \\
\hline
\end{tabular}


Table 3

Subject, terminal delay series (Delay Series), and AUC for each subject during the terminal delay series (Baseline), determination of the acute dose-response function (Control, VEH, 1.0, 3.0, and $10.0 \mathrm{mg} / \mathrm{kg}$ ), first five (First 5 Repeated) and last five (Last 5 Repeated) sessions of repeated administration of diazepam for 30-50 sessions, determination of the chronic dose-response function (VEH, 1.0, 3.0, and $10.0 \mathrm{mg} / \mathrm{kg}$ ), and baseline replication at the end of experimentation (Baseline 2).

\begin{tabular}{|c|c|c|c|c|c|c|c|}
\hline \multirow[b]{2}{*}{ Subject } & \multirow[b]{2}{*}{$\begin{array}{l}\text { Delay } \\
\text { Series } \\
\end{array}$} & \multirow[b]{2}{*}{ Baseline } & \multicolumn{5}{|c|}{ Acute Dose $(\mathrm{mg} / \mathrm{kg})$} \\
\hline & & & Control & VEH & 1.0 & 3.0 & 10.0 \\
\hline LEW-2 & 40 & 0.3604 & 0.3581 & 0.3924 & 0.3507 & 0.3993 & 0.6528 \\
\hline LEW-3 & 16 & 0.5475 & 0.4848 & 0.4115 & 0.5156 & 0.6641 & 0.8151 \\
\hline LEW-4 & 16 & 0.3688 & 0.3611 & 0.3984 & 0.3802 & 0.3594 & 0.4766 \\
\hline LEW-7 & 40 & 0.2792 & 0.3326 & 0.3423 & 0.3438 & 0.3385 & 0.3151 \\
\hline LEW-8 & 16 & 0.4000 & 0.4368 & 0.3188 & 0.3472 & 0.5078 & 0.3854 \\
\hline$M(S E M)$ & & $0.4041(0.04)$ & $0.4105(0.03)$ & $0.3969(0.02)$ & $0.4085(0.03)$ & $0.4376(0.05)$ & $0.4599(0.08)$ \\
\hline F344-1 & 16 & 0.5458 & 0.5331 & 0.3872 & 0.5443 & 0.3724 & 0.4005 \\
\hline F344-6 & 16 & 0.7417 & 0.7786 & 0.8167 & 0.6328 & 0.3464 & 0.1979 \\
\hline F344-7 & 40 & 0.6250 & 0.5317 & 0.5295 & 0.6563 & 0.5885 & 0.1615 \\
\hline F344-8 & 16 & 0.5625 & $--/--$ & --/-- & $--/--$ & $--/--$ & $--/--$ \\
\hline$M(S E M)$ & & $0.6141(0.04)$ & $0.5498(0.05)$ & $0.4911(0.06)$ & $0.5696(0.04)$ & $0.4730(0.04)$ & $0.2066(0.03)$ \\
\hline
\end{tabular}

Chronic Dose (mg/kg)

\begin{tabular}{|c|c|c|c|c|c|c|c|c|}
\hline Subject & $\begin{array}{l}\text { Delay } \\
\text { Series }\end{array}$ & $\begin{array}{c}\text { First } 5 \\
\text { Repeated } \\
\end{array}$ & $\begin{array}{c}\text { Last } 5 \\
\text { Repeated }\end{array}$ & VEH & 1.0 & 3.0 & 10.0 & Baseline 2 \\
\hline LEW-1 & 16 & 0.7792 & 0.6375 & 0.5025 & 0.6632 & 0.7917 & 0.7813 & 0.5125 \\
\hline LEW-3 & 16 & 0.5771 & 0.2167 & 0.0938 & 0.2188 & 0.2500 & 0.3423 & 0.3833 \\
\hline LEW-4 & 16 & 0.2750 & 0.5104 & 0.3646 & 0.2865 & 0.3438 & 0.3929 & 0.4000 \\
\hline LEW-5 & 16 & 0.2271 & 0.3604 & 0.3188 & 0.3021 & 0.3924 & 0.2642 & 0.3750 \\
\hline LEW-8 & 16 & 0.4104 & 0.4250 & 0.3576 & 0.3958 & 0.4640 & 0.4089 & 0.3479 \\
\hline$M(S E M)$ & & $0.4429(0.08)$ & $0.4390(0.07)$ & $0.3561(0.05)$ & $0.3486(0.06)$ & $0.4349(0.06)$ & $0.4278(0.06)$ & $0.3866(0.02)$ \\
\hline F344-1 & 16 & 0.3958 & 0.3271 & 0.3576 & 0.3698 & 0.4028 & 0.1797 & 0.6479 \\
\hline F344-2 & 16 & 0.5979 & 0.4000 & 0.2750 & 0.3672 & 0.4236 & 0.2240 & 0.2938 \\
\hline F344-7 & 40 & 0.2583 & 0.0979 & 0.1389 & 0.2083 & 0.0885 & 0.0885 & 0.1833 \\
\hline F344-8 & 16 & $--/--$ & $--/--$ & $--/--$ & $--/--$ & $--/--$ & $--/--$ & $--/--$ \\
\hline$M(S E M)$ & & $0.2663(0.08)$ & $0.2389(0.07)$ & $0.2241(0.04)$ & $0.2403(0.05)$ & $0.2471(0.07)$ & $0.1683(0.05)$ & $0.2945(0.08)$ \\
\hline
\end{tabular}



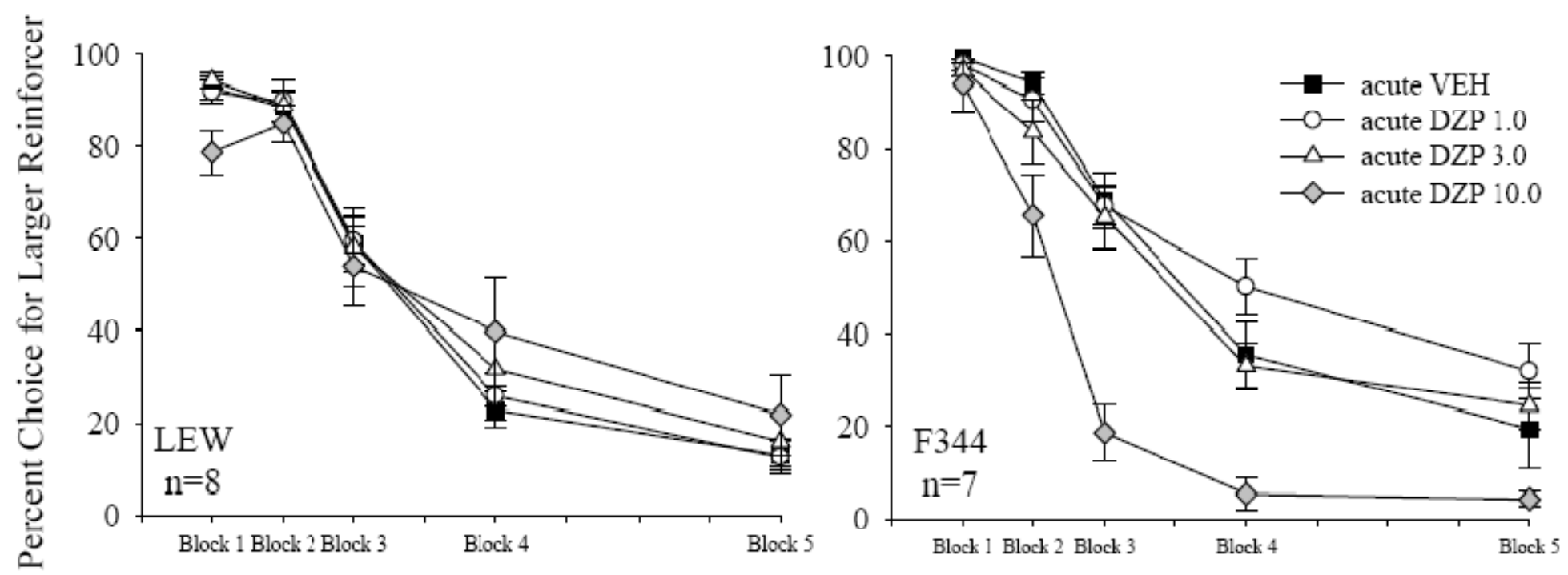

\section{Delay to Larger Reinforcer (s)}

Figure 3. Mean percent larger-reinforcer choice as a function of delay for acute diazepam administration, including vehicle (closed squares), 1.0 (open circles), 3.0 (open triangles), and 10.0 (gray diamonds) $\mathrm{mg} / \mathrm{kg}$ of diazepam for LEW (left panel) and F344 (right panel) rats. Error bars represent standard error of the mean. The $\mathrm{x}$-axis is composed of blocks rather than delay values as two LEW and three F344 rats responded in a 0, 5, 10, 20, and 40-s delay series, and six LEW and four F344 rats responded in a $0,2,4,8$, and 16-s delay series. 

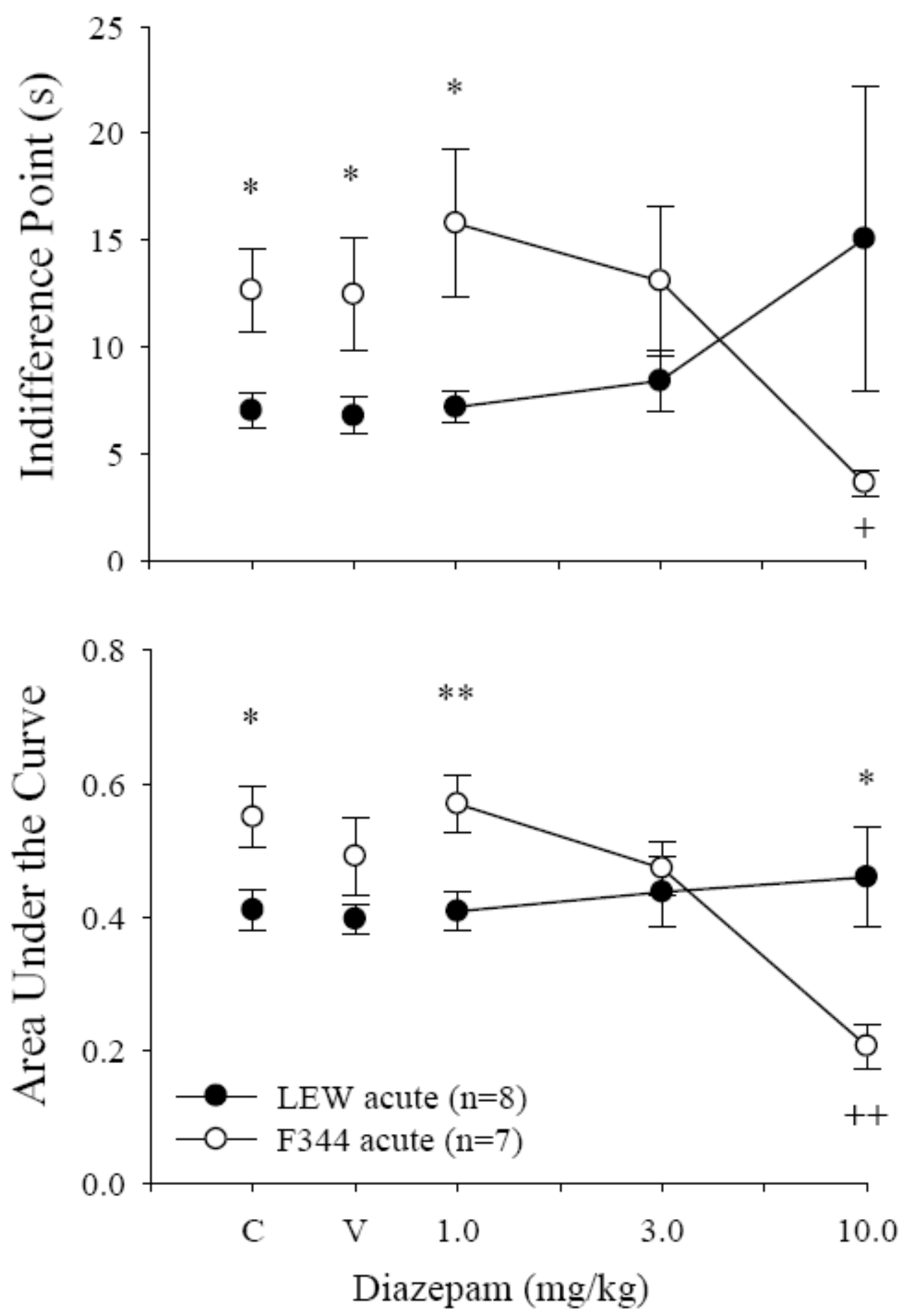

Figure 4. Mean indifference points (top panel) and AUC (bottom panel) as a function of doses of diazepam (control, vehicle, 1.0, 3.0, and $10.0 \mathrm{mg} / \mathrm{kg}$ ) during acute drug administration for LEW (closed symbols) and F344 (open symbols) rats. Error bars represent standard error of the mean. Single and double asterisks represent significance levels of $p<.05$ and $p<.01$, respectively, where differences occurred between rat strains at a particular dose. Single and double crosses represent significance levels of $p<.05$ and $p<.01$, respectively, where significant changes for a particular dose occurred relative to vehicle for each strain separately. 


\section{Repeated (Chronic) Drug Administration}

One LEW and one F344 rat did not receive repeated drug administration because the terminal delay series required a much longer period of time to determine compared to other subjects. Considering the lifespan of these rat strains, completing the repeated diazepam phase would likely not have been possible or healthy for these subjects. Analyses throughout the rest of the experiment are thus based on seven LEW and six F344 rats. Table 1 shows the chronic dose of diazepam administered and the number of sessions conducted during the repeated drug phase for individual subjects. There was no difference between the number of sessions required for LEW and F344 rats to complete the repeated drug phase.

Figure 5 shows mean percent larger-reinforcer choice for acute vehicle administration and the first and last five sessions of repeated diazepam administration (30-50 sessions) as a function of delay to the larger reinforcer (displayed as blocks of trials) for LEW (left panel) and F344 (right panel) rats (see Tables 2 and 3 for individual-subject data). Data from acute vehicle administration were included here to compare repeated drug effects to a non-drug condition. There was a differential effect of repeated diazepam administration between rat strains. For LEW rats, mean larger-reinforcer choice during repeated diazepam administration was similar to levels obtained during acute vehicle administration. For individual LEW rats however, indifference points and AUC often did change during the first five sessions of repeated administration, however, the effects were not systematic. For F344 rats, larger-reinforcer choice during repeated diazepam administration decreased relative to acute vehicle administration. Within the first five sessions of repeated diazepam administration, there was an overall decrease in larger-reinforcer choice for four of six F344 rats. For individual subjects, the observed decrease in larger-reinforcer choice only occurred for F344 rats that received the largest doses 
(5.6 and $10.0 \mathrm{mg} / \mathrm{kg}$ ) of diazepam, and a slight increase across the first five sessions of repeated diazepam occurred for the other F344 rats that received the smallest dose $(1.0 \mathrm{mg} / \mathrm{kg})$ of diazepam repeatedly.

For both rat strains, there was little change in percent larger-reinforcer choice from the first five to the last five sessions of repeated diazepam administration. For individual subjects, indifference points and AUC often did change from the first to the last five sessions of this phase, however, the effects were not systematic. When combined, any effects that occurred for individual subjects were not apparent in the averages. Overall, across repeated diazepam administration, mean percent larger-reinforcer choice remained unchanged for LEW rats and for F344 rats, decreased within the first five sessions and remained low across the repeated phase. The mean AUC during the last five sessions of repeated diazepam administration for F344 rats was nearly significantly smaller relative to acute vehicle administration $[F(1,5)=6.49, p=.051]$. 

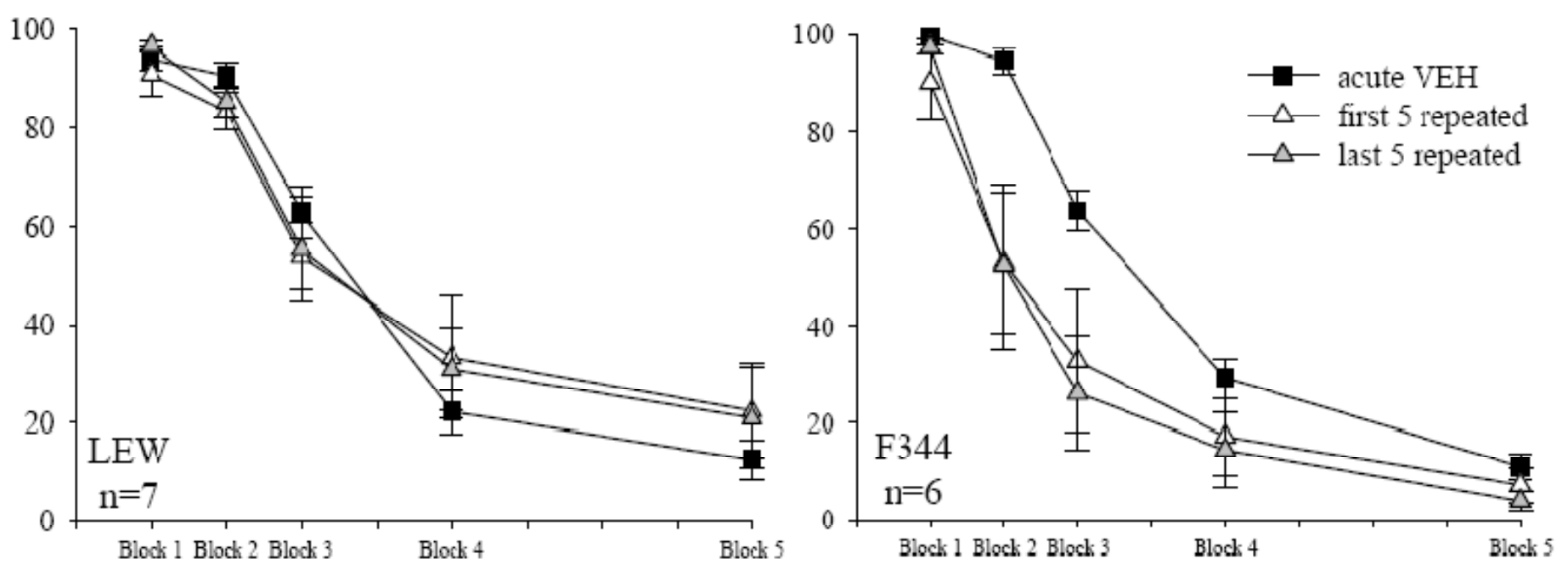

Delay to Larger Reinforcer (s)

Figure 5. Mean percent larger-reinforcer choice as a function of delay during acute vehicle administration (closed squares) and the first (open triangles) and last (gray triangles) five sessions of repeated diazepam administration. Error bars represent standard error of the mean. The X-axis is composed of blocks as one LEW and three F344 rats responded in a 0, 5, 10, 20, and 40-s delay series, and six LEW and three F344 rats responded in a 0, 2, 4, 8, and 16-s delay series. 


\section{Determination of the Chronic Dose-Response Function}

Table 1 shows the number of sessions conducted during determination of chronic doseresponse functions for individual subjects. There was no difference between the number of sessions required for LEW and F344 rats to complete this phase. Figure 6 shows mean percent larger-reinforcer choice for vehicle and each dose of repeated diazepam as a function of delay to the larger reinforcer (displayed as blocks of trials) for LEW (left panel) and F344 (right panel) rats during determination of the chronice dose-response function. For both rat strains, mean percent larger-reinforcer choice did not change systematically for any chronic dose of diazepam administered.

The above findings are also indicated in Figure 7, which shows indifference points (top panels) and AUC (bottom panels) for LEW (left panels) and F344 (right panels) rats as a function of acute and chronic doses of diazepam (see Tables 2 and 3 for individual-subject data). Again, for LEW rats, there was no effect of dose on indifference points or AUC during determination of the chronic dose-response function, and indifference points and AUC during chronic diazepam administration were not different from those obtained during acute diazepam administration. The large increases that had occurred for LEW-2 and LEW-3 during acute administration of the $10.0 \mathrm{mg} / \mathrm{kg}$ dose of diazepam no longer occurred when it was administered during the chronic dose-response determinations.

For F344 rats, there was an overall decrease in larger-reinforcer choice from the acute to repeated diazepam phases as indicated by an overall decrease in indifference points and AUC across all doses of diazepam administered (see Figure 7). When vehicle was administered (nondrug sessions), AUC for F344 rats decreased relative to acute vehicle administration $[F(1,5)=$ $16.71, p<.01]$. Indifference points and AUC for F344 rats also decreased significantly from 
acute to chronic administration of $1.0[F(1,5)=8.31, p<.05 ; F(1,5)=68.65, p<.01$, respectively $]$ and $3.0 \mathrm{mg} / \mathrm{kg}[F(1,5)=6.59, p=.05 ; F(1,5)=8.99, p<.05$, respectively $]$ of diazepam. Indifference points and AUC at the largest dose administered $(10.0 \mathrm{mg} / \mathrm{kg})$ remained low across the acute and chronic diazepam conditions. Considering these results in combination with the decreases in larger-reinforcer choice within the first five sessions of repeated diazepam administration, a baseline shift occurred for F344 rats that remained throughout all repeated phases of diazepam administration. 

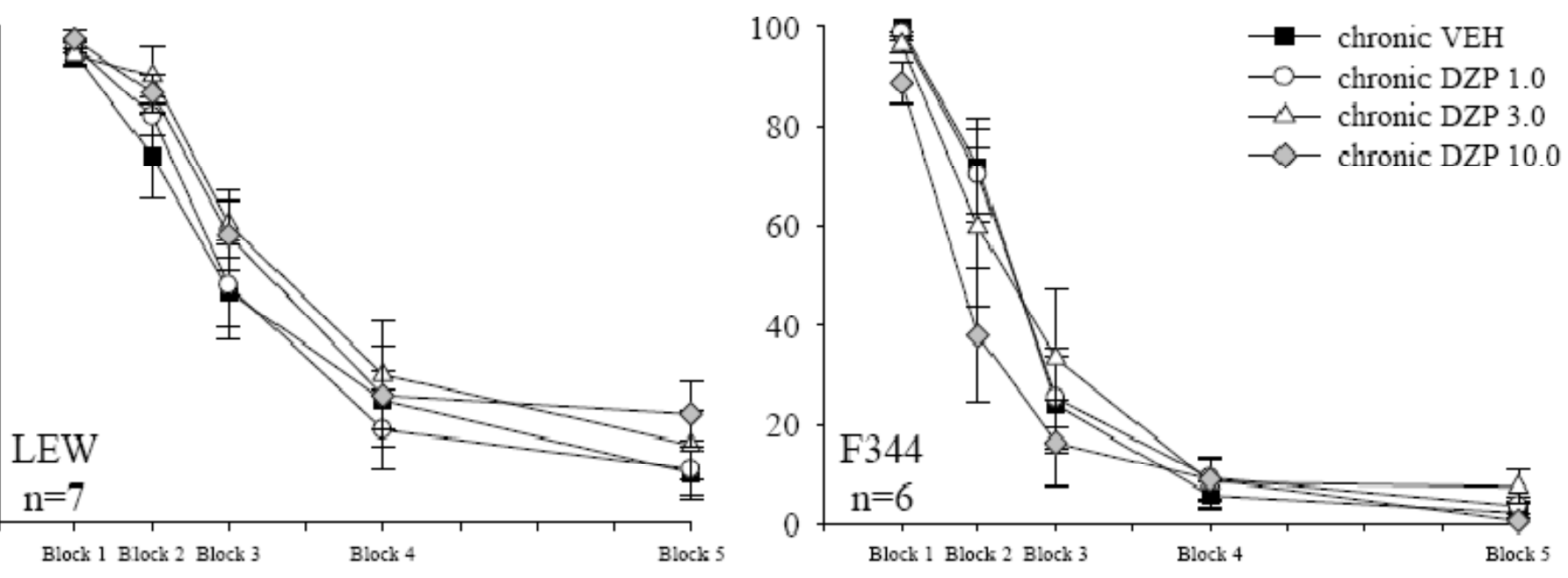

Delay to Larger Reinforcer (s)

Figure 6. Mean percent larger-reinforcer choice as a function of delay during determination of the chronic dose-response function, including vehicle (closed squares), 1.0 (open circles), 3.0 (open triangles), and 10.0 (gray diamonds) $\mathrm{mg} / \mathrm{kg}$ of diazepam for LEW (left panel) and F344 (right panel) rats. Error bars represent standard error of the mean. The x-axis is composed of blocks rather than delay values as one LEW and three F344 rats responded in a 0, 5, 10, 20, and 40-s delay series, and six LEW and three F344 rats responded in a 0, 2, 4, 8, and 16-s delay series. 

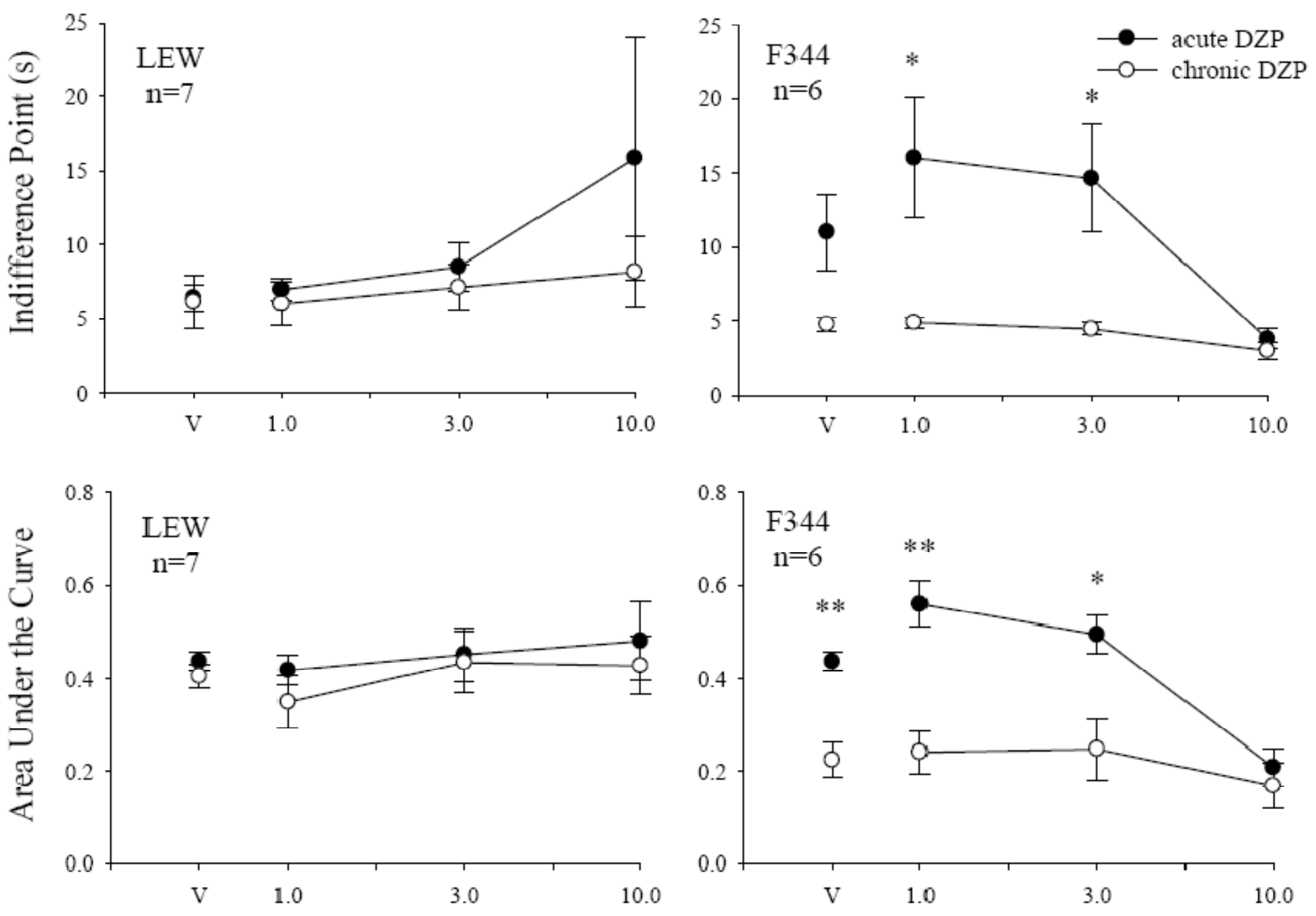

\section{Diazepam (mg/kg)}

Figure 7. Mean indifference points (top panels) and AUC (bottom panels) as a function of doses of diazepam (vehicle, 1.0, 3.0, and $10.0 \mathrm{mg} / \mathrm{kg}$ ) during acute (closed symbols) and chronic (open symbols) drug administration for LEW (left panels) and F344 (right panels) rats. Error bars represent standard error of the mean. Single and double asterisks represent significance levels of $p<.05$ and $p<.01$, respectively, where differences occurred between acute and chronic administration at a particular dose. 


\section{Baseline Replication}

Table 1 shows the number of sessions required to meet criterion $(80 \%$ larger-reinforcer choice across all blocks of trials) during 0 -s probe sessions after completion of chronic drug administration and number of sessions conducted during the baseline replication condition for individual subjects as well as means and standard error of the mean for each strain separately. There was no difference between the number of sessions for LEW and F344 rats to meet the criterion during 0 -s probe sessions or to reach stability during baseline replication.

Figure 8 shows mean percent larger-reinforcer choice as a function of delay to the larger reinforcer (displayed as blocks of trials) for only those LEW (left panel) and F344 (right panel) rats that completed the chronic drug phase. Choice data are from the last five sessions of the terminal baseline phase conducted at the beginning of experimentation (prior to drug administration) and the last five sessions of the baseline replication condition during which drug administration had ceased. During the initial baseline phase, LEW rats chose the largerreinforcer less than F344 rats. This was indicated by shorter indifference points and smaller AUC for LEW rats. Upon the baseline replication, the differences in larger-reinforcer choice were no longer present (see Tables 2 and 3 for individual-subject data). Similar rates of discounting occurred for LEW and F344 rats, and this was indicated by similar indifference points and AUC during the baseline replication phase for each strain. Throughout experimentation, indifference points and AUC remained relatively unchanged for LEW rats, while indifference points were initially longer and AUC were initially larger for F344 rats. Early in the repeated administration phase, indifference points and AUC for F344 rats decreased and remained at low levels throughout experimentation. 

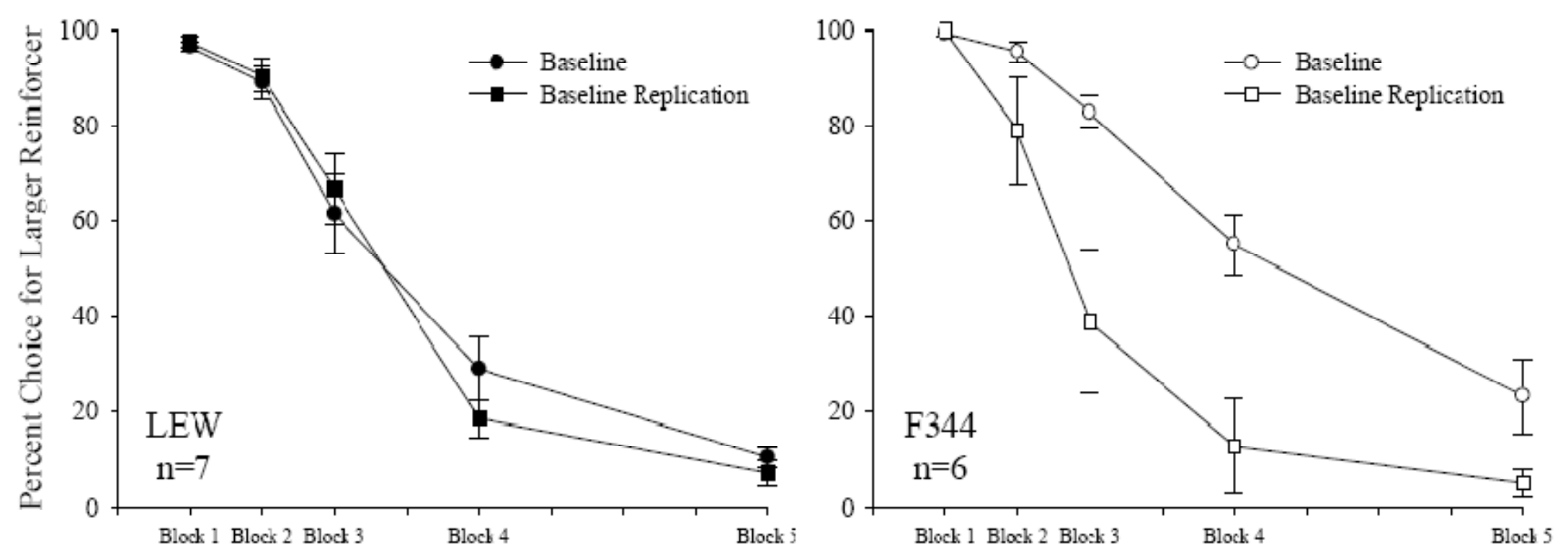

\section{Delay to Larger Reinforcer (s)}

Figure 8. Mean percent larger-reinforcer choice as a function of delay blocks for the last five sessions conducted during the terminal (baseline) delay series (circles) and last five sessions from the baseline replication phase (squares) for only those LEW (left panel) and F344 (right panel) rats that completed all phases of the experiment. Error bars represent standard error of the mean. The $\mathrm{x}$-axis is composed of blocks rather than delay values as one LEW and three F344 rats responded in a $0,5,10,20$, and 40-s delay series, and six LEW and three F344 rats responded in a $0,2,4,8$, and 16-s delay series. 


\section{Discussion}

Consistent with previous research (e.g., Anderson \& Woolverton, 2005; Madden et al., 2008), delay discounting was observed for both rat strains in that, as delay to the larger reinforcer increased, choice for that alternative decreased. Delay series for individual subjects were functionally determined and, despite obtaining steeper discounting functions and smaller AUC for LEW rats, intermediate functions were obtained for both rat strains. LEW rats had shorter indifference points compared to F344 rats, and this finding is consistent with previous research using the same discounting procedure where delays systematically increased within sessions (Anderson \& Woolverton; Anderson \& Diller, 2010; Garcia-Lecumberri et al., 2010; Huskinson et al., revisions submitted) and using a different procedure where delays systematically increased between sessions (Madden et al., 2008). Despite significant differences in mean indifference points at baseline, there was some overlap between strains. For example, relatively long indifference points were observed for two LEW rats that were similar to the four shortest indifference points observed for F344 rats.

In the current study, LEW rats required more 0 -s probe sessions during the terminal (baseline) delay series compared to F344 rats. It is possible that LEW rats were less sensitive to differences in reinforcer amount, and this could have contributed to differences in baseline rates of delay discounting. However, differences in the number sessions required to pass 0 -s probe sessions were no longer present during the acute phase, yet rates of discounting were steeper for LEW compared to F344 rats during non-drug sessions (vehicle and control sessions). It is also possible that sensitivity to reinforcer delay is different for LEW compared to F344 rats. The procedure used, however, cannot separate effects of reinforcer amount and delay, and strain differences in discounting may result from differential control by reinforcer amount, delay, or 
both. Neurochemical differences also could have contributed to differences in baseline rates of delay discounting between these rat strains. In general, LEW rats have lower levels of DA and 5-HT in various brain regions relative to F344 rats (Burnet et al., 1992; Flores et al., 1998; Selim \& Bradberry, 1996), and depletion of these neurotransmitters is related to steeper rates of discounting (Kheramin et al., 2004; Mobini et al., 2000; Wogar et al., 1993). It is important to note that neurochemical measures were not used in the present experiment, however, future research correlating delay-discounting rates and neurotransmitter levels could provide empirical support for this relation.

During the acute drug phase, steeper rates of discounting continued to occur for LEW relative to F344 rats during control and vehicle sessions, thus, the baseline differences persisted throughout the acute diazepam phase. For F344 rats, mean indifference points and AUC did decrease slightly during control and vehicle sessions relative to those obtained at baseline, and this effect did not occur for LEW rats. It is possible that larger-reinforcer choice gradually declines with extended exposure to the task, particularly for subjects with initially shallower discounting functions. In the current study, the two F344 rats with the longest indifference points and largest AUC (F344-3 and F344-7) showed the greatest decrease from baseline to control sessions. If extended exposure to the discounting task was responsible for the observed decreases in larger-reinforcer choice for F344 rats, it would be likely that this effect would also occur for LEW rats.

The finding that acute diazepam did not alter larger-reinforcer choice in LEW rats is consistent with other research examining effects of acute diazepam administration on different behavioral measures in LEW rats. Takahashi and colleagues (2001) examined effects of different doses of diazepam on an elevated plus-maze task in LEW rats relative to spontaneously 
hypertensive rats (SHR). Doses that affected behavior in SHRs had no effect on behavior in LEW rats. LEW rats were also less sensitive to behavioral effects of chlordiazepoxide, a benzodiazepine, on a successive negative-contrast procedure compared to F344 rats (Freet, Tesche, Tompers, Riegel, \& Grigson, 2006). Within the same procedure, chlordiazepoxide increased overall consumption of a sucrose solution for F344, but not LEW rats (Freet et al., 2006).

Perhaps delay discounting is another measure that, in LEW rats, is not sensitive to effects of benzodiazepines. Examination of individual-subject data suggested otherwise. Acute diazepam did not affect mean larger-reinforcer choice in LEW rats when effects of each dose were compared separately, but different doses of diazepam did have effects for individual subjects. For example, at least one of the doses administered increased AUC in LEW rats relative to vehicle, but which dose had this effect was variable across subjects and was obscured by presenting only the means for each dose. Increased larger-reinforcer choice in LEW rats is consistent with Evenden and Ryan's (1996) findings that diazepam increased larger-reinforcer choice in Sprague-Dawley rats. In studies that report no effect (Freet et al., 2006; Takahashi et al., 2001), it is possible that individual LEW rats were insensitive to benzodiazepine administration or that effects were observed for individual LEW rats that were obscured by reporting only the group means. This highlights the importance of reporting individual-subject data, particularly in experiments that use small-n designs.

For F344 rats, at least one of the two smallest doses $(1.0$ and $3.0 \mathrm{mg} / \mathrm{kg})$ of diazepam increased indifference points and AUC for all but one F344 rat, and this is also consistent with Evenden and Ryan's (1996) results with diazepam in Sprague-Dawley rats. Conversely, the largest dose of diazepam decreased larger-reinforcer choice in F344 rats, and this is consistent 
with Cardinal et al.'s (2000) finding that relatively large doses of chlordiazepoxide decrease larger-reinforcer choice in Listar hooded rats. Evenden and Ryan and Cardinal et al. are used here as a comparison, because both studies used a delay-discounting procedure that was similar to the one used in the present study. A majority of the research examining effects of benzodiazepines on delay discounting have used a T-maze procedure. Comparisons between the current study and those using a T-maze procedure are more difficult because there are many differences in procedural variables.

Within the type of procedure used in the current study and also by Evenden and Ryan (1996) and Cardinal et al. (2000), it is possible that discrepant findings with benzodiazepines and delay discounting result from differential sensitivities to different doses of diazepam in different rat strains and different subjects within a given rat strain. It seems that an increase in largerreinforcer choice, despite occurring at different doses for different subjects is a systematic effect worth noting. When experimenters do not report individual-subject data, at least for small-n designs, it is not clear whether an effect did not occur, or whether an effect did occur but was observed at different doses for different subjects and was obscured by reporting group means only. Neither Evenden and Ryan nor Cardinal et al. report individual-subject data.

It is also possible that discrepant findings with benzodiazepines and delay discounting result from administration of an insufficient range of doses. For example, Evenden and Ryan (1996) found increases in larger-reinforcer choice following smaller doses of diazepam (0.3 and $1.0 \mathrm{mg} / \mathrm{kg}$ ). Other doses of diazepam were not tested. Cardinal et al. (2000) found decreases in larger-reinforcer choice following administration of the largest dose of chlordiazepoxide tested $(10.0 \mathrm{mg} / \mathrm{kg})$. These researchers used multiple doses, but did not examine effects of doses 
smaller than $1.0 \mathrm{mg} / \mathrm{kg}$. The present experiment found increases and decreases in largerreinforcer choice depending on strain and dose of diazepam.

The increases in larger-reinforcer choice observed for individual LEW and F344 rats following acute diazepam administration may have resulted from benzodiazepine's ability to enhance consumption and palatability of food (see Cooper, 2004 for a review). If diazepam functioned to enhance palatability of food, it may have also increased control of choice by reinforcer amount. It cannot be ruled out, however, that diazepam altered control of choice by reinforcer delay or both amount and delay. Again, based on the procedures used in the current study, it is not possible to separate drug effects on choice controlled by differences in reinforcer amount and reinforcer delay (Pitts \& Febbo, 2004).

The decreases in larger-reinforcer choice observed for F344 rats following acute diazepam administration may be related to effects of benzodiazepine administration on DA and 5-HT. For example, benzodiazepines decreased extracellular levels of DA and 5-HT neuronal activity in specific brain regions (Finlay et al., 1995; Stein et al., 1975). Administration of the largest dose may have resulted in lower levels of DA and 5-HT that contributed to steeper rates of discounting in F344 rats. Larger-reinforcer choice did not decrease for LEW rats following acute administration of diazepam. LEW rats already have lower levels of DA and 5-HT, and it is possible that a floor effect occurred with these rats. Again, neurochemical measures were not taken in the current study, and future research could correlate DA and 5-HT levels with rates of discounting following benzodiazepine administration.

During the repeated diazepam phase, there were no systematic effects for LEW rats when larger-reinforcer choice was compared during a non-drug condition (acute vehicle) to the first five sessions of repeated administration. The dose administered during the repeated phase 
(chronic dose) was functionally determined for the greatest change in choice, and overall, was different for individual subjects. For two LEW rats, this dose initially decreased largerreinforcer choice when administered during the acute phase, and for five LEW rats, this dose initially increased larger-reinforcer choice during the acute phase. If only those doses that increased larger-reinforcer choice (or vice versa) were chosen as the repeated dose for all subjects, more systematic effects may have occurred. Because it was unclear during the acute phase whether diazepam would increase, decrease, or have no effect on larger-reinforcer choice, it was not possible to set this type of criterion in advance. This could also have resulted in failure to find systematic changes in larger-reinforcer choice for LEW rats from the first five to the last five sessions of the repeated phase.

For F344 rats, larger-reinforcer choice did decrease during the first five sessions of the repeated phase in the four F344 rats that received the largest doses of diazepam as their repeated dose. With these subjects, larger-reinforcer choice decreased rapidly and remained low across the repeated phase. By the end of the repeated phase, larger-reinforcer choice had decreased relative to acute vehicle for all six F344 rats. That more systematic effects occurred for F344 relative to LEW rats is consistent with other studies examining repeated exposure to diazepam in LEW and F344 rats. For example, LEW rats show fewer signs of physical dependence and withdrawal compared to F344 rats following repeated exposure and removal of food mixed with diazepam (Suzuki et al., 1992). This difference in physical dependence occurred despite having similar blood-diazepam levels across both strains.

Compared to acute administration of diazepam, chronic diazepam administration had no effect on mean percent larger-reinforcer choice for LEW rats. Indifference points and AUC across all doses were similar to those obtained during both acute and chronic vehicle 
administration. However, the variability in indifference points and AUC across subjects decreased for LEW rats following chronic diazepam administration. For F344 rats, the overall decrease in larger-reinforcer choice that occurred during the first five days of repeated diazepam administration persisted during determination of the chronic dose-response functions. Across all doses administered, including vehicle, indifference points and AUC were similar to those obtained following acute administration of the largest dose of diazepam. Following many weeks (and months by the end of this phase) of daily diazepam dosing, levels of DA and 5-HT may have decreased to levels that could not recover when vehicle or smaller doses of diazepam were administered.

During the baseline replication phase, when all injections ceased, the rate of discounting for LEW rats was similar to the rate of discounting observed for these rates during the initial baseline condition. Thus, delay discounting in LEW rats remained, at least on average, relatively unchanged across multiple conditions of the experiment. For F344 rats, the overall decrease in larger-reinforcer choice observed early in repeated diazepam exposure, persisted into the baseline replication phase. It seems that the baseline shift in F344 rats could have occurred for at least three reasons. First, repeated diazepam could have resulted in a relatively permanent baseline shift in larger-reinforcer choice. Rats that completed repeated conditions of diazepam administration were exposed to daily diazepam administration for two to three months. If the initial baseline shift that occurred within the first five sessions of repeated administration was not relatively permanent, one might expect larger-reinforcer choice to return to baseline levels as individual subjects became tolerant to diazepam's effects. This did not occur throughout repeated administration nor did rates of discounting return to initial baseline levels following a minimum of 20 sessions on the delay-discounting task when drug was no longer administered. 
The second and third potential reasons for an overall decrease in larger-reinforcer choice for F344 rats could have been repeated exposure to the delay-discounting task and subject maturation, respectively. Exposure to diazepam across the multiple conditions of the experiment, exposure to the discounting task, and subject maturation were confounded throughout experimentation. It is possible that as F344 rats aged or experienced continued exposure to the task, larger-reinforcer choice would have decreased anyway. However, the finding that aged F344 rats have shallower discounting functions compared to young F344 rats, using a similar procedure to the one used in the current study, would predict the opposite (Simon et al., 2010). The findings from Simon and colleagues do not help to rule out extended exposure to the discounting task as a potential contributor to decreases in larger-reinforcer choice as both aged and young F344 rats were exposed to the task for a short period of time. That the same overall decrease in larger-reinforcer choice did not occur for LEW rats, however, makes effects of both age and exposure to the task less plausible.

The present study is the first to report acute and repeated effects of diazepam on delay discounting in LEW and F344 rats. LEW and F344 rats have behavioral and neurochemical differences that are correlated with baseline rates of delay discounting. Acute administration of diazepam had differential effects on larger-reinforcer choice in LEW and F344 rats and for individual subjects within each strain. Repeated administration of diazepam did not systematically affect larger-reinforcer choice in LEW rats and resulted in an overall decrease in larger-reinforcer choice for F344 rats that persisted throughout a baseline replication phase. It seems that the results discussed above have raised more questions about, and potential directions for future investigation of, multiple environmental, genetic, and neurochemical variables involved in delay discounting and effects of benzodiazepines on delay discounting. 


\section{References}

Acheson, A., Reynolds, B., Richards, J. B., \& de Wit, H. (2006). Diazepam impairs behavioral inhibition but not delay discounting or risk taking in healthy adults. Experimental and Clinical Psychopharmacology, 14, 190-198.

Ainslie, G. (1975). Specious reward: A behavioral theory of impulsiveness and impulse control. Psychological Bulletin, 82, 463-496.

Anderson, K. G., \& Diller, J. W. (2010). Effects of acute and repeated nicotine administration on delay discounting in Lewis and Fischer 344 rats. Behavioural Pharmacology, 21, 754764.

Anderson, K. G., \& Elcoro, M. (2007). Response acquisition with delayed reinforcement in Lewis and Fischer 344 rats. Behavioural Processes, 74, 311-318.

Anderson, K. G., \& Woolverton, W. L. (2005). Effects of clomipramine on self-control choice in Lewis and Fischer 344 rats. Pharmacology, Biochemistry and Behavior, 80, 387-393.

Bickel, W. K., Odum, A. L., \& Madden, G. J. (1999). Impulsivity and cigarette smoking: Delay discounting in current, never, and ex-smokers. Psychopharmacology, 146, 447-454.

Bizot, J. C., Bihan, C. L., Puech, A. J., Hamon, M., \& Theibot, M. H. (1999). Serotonin and tolerance to delay of reward in rats. Psychopharmacology, 146, 400-412.

Burnet, P. W., Mefford, I. N., Smith, C. C., Gold, P. W., \& Sternberg, E. M. (1992). Hippocampal 8-[3H]Hydroxy-2-(Di-n-Propylamino) tetralin binding site densities, serotonin receptor $\left(5-\mathrm{HT}_{1 \mathrm{~A}}\right)$ messenger ribonucleic acid abundance, and serotonin levels parallel the activity of the hypothalamopituitary-adrenal axis in rat. Journal of Neurochemistry, 59(3), 1062-1070.

Cardinal, R. N., Robbins, T. W., \& Everitt, B. J. (2000). The effects of $d$-amphetamine, 
chlordiazepoxide, $\alpha$-flupenthixol and behavioural manipulations on choice of signaled and unsignalled delayed reinforcement in rats. Psychopharmacology, 152, 362-375.

Cardinal, R. N., Robbins, T. W., \& Everitt, B. J. (2003). Choosing delayed rewards: Perspectives from learning theory, neurochemistry, and neuroanatomy. In: N. Heather, \& R. Vuchinich (Eds.), Choice, behavioral economics and addiction (pp. 183-213). Amsterdam: Elsevier.

Charrier, D., \& Thiebot, M. H. (1996). Effects of psychotropic drugs on rat responding in an operant paradigm involving choice between delayed reinforcers. Pharmacology, Biochemistry and Behavior, 54(1), 149-157.

Christensen, C. J., Kohut, S. J., Handler, S., Silberberg, A., Riley, A. L. (2009). Demand for food and cocaine in Fischer and Lewis rats. Behavioral Neuroscience, 123, 165-171.

Coffey, S. F., Gudleski, G. D., Saladin, M. E., \& Brady, K. T. (2003). Impulsivity and rapid discounting of delayed hypothetical rewards in cocaine-dependent individuals. Experimental Clinical Psychopharmacology, 11, 18-25.

Cooper, S. J. (2004). Endocannabinoinds and food consumption: Comparisons with benzodiazepine and opioid palatability-dependent appetite. European Journal of Pharmacology, 500, 37-49.

Diergaarde, L., Pattij, T., Poortvliet, I., Hogenboom, F., de Vries, W., Schoffelmeer, A. N. M., \& de Vries, T. J. (2008). Impulsive choice and impulsive action predict vulnerability to distinct stages of nicotine seeking in rats. Biological Psychiatry, 63, 301-308.

Diller, J. W., Saunders, B. T., \& Anderson, K. G. (2008). Effects of acute and repeated administration of caffeine on temporal discounting in rats. Pharmacology, Biochemistry, and Behavior, 89, 546-555. 
Doyon, W. M., Anders, S. K., Ramachandra, V. S., Czachowski, C. L., \& Gonzales, R. A. (2005). Effect of operant self-administration of $10 \%$ ethanol plus $10 \%$ sucrose on dopamine and ethanol concentrations in the nucleus accumbens. Journal of Neurochemistry, 93, 1469-1481.

Evenden, J. L. (1999). Varieties of impulsivity. Psychopharmacology, 146, 348-361.

Evenden, J. L., \& Ryan, C. N. (1996). The pharmacology of impulsive behavior in rats: The effects of drugs on response choice with varying delays of reinforcement. Psychopharmacology, 128, 161-170.

Evenden, J. L., \& Ryan, C. N. (1999). The pharmacology of impulsive behavior in rats VI: The effects of ethanol and selective serotonergic drugs on response choice with varying delays of reinforcement. Psychopharmacology, 146, 413-421.

Finlay, J. M., Zigmond, M. J., \& Abercrombie, E. D. (1995). Increased dopamine and norepinephrine release in medial prefrontal cortex induced by acute and chronic stress: Effects of diazepam. Neuroscience, 64, 619-628.

Flores, G., Wood, G. K., Barbeau, D., Quirion, R., \& Srivastava, K. L. (1998). Lewis and Fischer rats: A comparison of dopamine transporter and receptors levels. Brian Research, 814, $34-40$.

Freeman, K. B., Kearns, D. N., Kohut, S. J., Riley, A. L. (2009). Strain differences in patterns of drug-intake during prolonged access to cocaine self-administration. Behavioral Neuroscience, 123, 156-164.

Freet, C. S., Tesche, J. D., Tompers, D. M., Riegel, K. E., \& Grigson, P. S. (2006). Lewis rats are more sensitive than Fischer rats to successive negative contrast, but less sensitive to the anxioltic and appetite-stimulating effects of chlordiazepoxide. Pharmacology, 
Biochemistry, and Behavior, 85, 378-384.

Garcia-Lecumberri, C., Torres, I., Martin, S., Crespo, J. A., Miguens, M., Nicanor, C., et al. (2010). Strain differences in the dose-response relationship for morphine selfadministration and impulsive choice between Lewis and Fischer 344 rats. Journal of Psychopharmacology, doi: 10.1177/0269881110367444.

Hoffman, W. F., Moore, M., Templin, R., McFarland, B., Hitzemann, R. J., Mitchell, S. H. (2006). Neuropsychological function and delay discounting in methamphetaminedependent individuals. Psychopharmacology, 188, 162-170.

Huskinson, S. L., Krebs, C. A., \& Anderson, K. G. (revisions submitted). Effects of acute and repeated administration of $d$-amphetamine on delay discounting in Lewis and Fischer 344 rats. Psychopharmacology.

Kheramin, S., Body, S., Ho, M. Y., Velazquez-Martinez, D. N., Bradshaw, C. M., Szabadi, E., et al. (2004). Effects of orbital prefrontal cortex dopamine depletion on inter-temporal choice: A quantitative analysis. Psychopharmacology, 175, 206-214.

Kollins, S. H. (2003). Delay discounting is associated with substance use in college students. Addictive Behaviors, 28, 1167-1173.

Kosten, T. A., Miserendino, M. J. D., Haile, C. C., DeCaprio, J. L., Jatlow, P. I. \& Nester, E. J. (1997). Acquisition and maintenance of intravenous cocaine self-administration in Lewis and Fischer inbred rat strains. Brain Research, 778, 418-429.

Kosten, T. A., Zhang, X. Y., Haile, C. N. (2007). Strain differences in maintenance of cocaine self-administration and their relationship to novelty activity responses. Behavioral Neuroscience, 121, 380-388.

Lobo, I. A., \& Harris, R. A. (2008). GABA $\mathrm{A}_{\mathrm{A}}$ receptors and alcohol. Pharmacology, Biochemistry 
and Behavior, 90, 90-94.

Madden, G. J., Bickel, W. A., Jacobs, E. A. (1999). Discounting of delayed rewards in opioiddependent outpatients: Exponential or hyperbolic discounting functions? Experimental and Clinical Psychopharmacology, 7(3), 284-293.

Madden, G. J., Petry, N. M., Badger, G. J., \& Bickel, W. K. (1997). Impulsive and self-control choices in opioid-dependent patients and non-drug-using control participants: Drug and monetary reward. Experimental and Clinical Psychopharmacology, 5(3), 256-262.

Madden, G. J., Smith, N. G., Brewer, A. T., Pinkston, J. W., \& Johnson, P. S. (2008). Steadystate assessment of impulsive choice in Lewis and Fischer 344 rats: Between-condition delay manipulations. Journal of the Experimental Analysis of Behavior, 90, 333-344.

Mazur, J. E. (1987). An adjusting procedure for studying delayed reinforcement. In: M. L. Commons, J. E. Mazur, J. A. Nevin, \& H. Rachlin (Eds.), Quantitative analysis of behavior: The effect of delay and of intervening events on reinforcement value (pp. 5573). Hillsdale, NJ: Erlbaum.

Mitchell, S. H. (1999). Measures of impulsivity in cigarette smokers and non-smokers. Psychopharmacology, 146, 455-464.

Mobini, S., Chiang, T. J., Al-Ruwaitea, A. S., Ho, M. Y., Bradshaw, C. M., \& Szabadi, E. (2000). Effect of central 5-hydroxytryptamine depletion on inter-temporal choice: A quantitative analysis. Psychopharmacology, 149, 313-318.

Mocsary, Z., \& Bradberry, C. W. (1996). Effect of ethanol on extracellular dopamine in nucleus accumbens: Comparison between Lewis and Fischer 344 rat strains. Brain Research, 706, 194-198.

Monterosso, J. R., Ainslie, G., Xu, J., Cordova, X., Domier, C. P., London, E. D. (2007). 
Frontoparietal cortical activity of methamphetamine-dependent and comparison subjects performing a delay discounting task. Human Brain Mapping, 28, 383-393.

Myerson, J., \& Green, L. (1995). Discounting of delayed rewards: Models of individual choice. Journal of the Experimental Analysis of Behavior, 64, 263-276.

Myerson, J., Green, L., \& Warusawitharana, M. (2001). Area under the curve as a measure of discounting. Journal of the Experimental Analysis of Behavior, 76, 235-243.

Olmstead, M. C., Hellemans, K. G., \& Paine, T. A. (2006). Alcohol-induced impulsivity in rats: an effect of cue salience? Psychopharmacology, 184, 221-228.

Ortner, C. N., MacDonald, T. K., \& Olmstead, M. C. (2003). Alcohol intoxication reduces impulsivity in the delay-discounting paradigm. Alcohol \& Alcoholism, 38(2), 151-156.

Perry, J. L., \& Carroll, M. E. (2008). The role of impulsive behavior in drug abuse. Psychopharmacology, 200, 1-26.

Perry, J. L., Larson, E. B., German, J. P., Madden, G. J., \& Carroll, M. E. (2005). Impulsivity (delay discounting) as a predictor of acquisition of IV cocaine self-administration in female rats. Psychopharmacology, 178, 193-201.

Perry, J. L., Nelson, S. E., \& Carroll, M. E. (2008). Impulsive choice as a predictor of acquisition of IV cocaine self-administration and reinstatement of cocaine-seeking behavior in male and female rats. Experimental and Clinical Psychopharmacology, 16, 165-177.

Perry, J. L., Stairs, D. J., \& Bardo, M. T. (2008). Impulsive choice and environmental enrichment: Effects of $d$-amphetamine and methylphenidate. Behavioural Brain Research, 193, 48-54.

Petry, N. M. (2001). Delay discounting of money and alcohol in actively using alcoholics, currently abstinent alcoholics, and controls. Psychopharmacology, 154, 243-250. 
Pitts, R. C., \& Febbo, S. M. (2004). Quantitative analyses of methamphetamine's effects on selfcontrol choices: Implications for elucidating behavioral mechanisms of drug action. Behavioural Processes, 66, 213-233.

Poulos, C. X., Le, A. D., \& Parker, J. L. (1995). Impulsivity predicts individual susceptibility to high levels of alcohol self-administration. Behavioural Pharmacology, 6, 810-814.

Poulos, C. X., Parker, J. L., \& Le, A. D. (1996). Dexfenfluramine and 8-OH-DPAT modulate impulsivity in a delay-of-reward paradigm: Implications for a correspondence with alcohol consumption. Behavioural Pharmacology, 7, 395-399.

Reynolds, B. (2006). A review of delay-discounting research with humans: Relations to drug use and gambling. Behavioural Pharmacology, 17(8), 651-667.

Reynolds, B., Richards, J. B., Dassinger, M., \& de Wit, H. (2004). Therapeutic doses of diazepam do no alter impulsive behavior in humans. Pharmacology, Biochemistry and Behavior, 79, 17-24.

Reynolds, B., Richards, J. B., Horn, K., \& Karraker, K. (2004). Delay discounting and probability discounting as related to cigarette smoking status in adults. Behavioural Processes, 65, 35-42.

Richards, J. B., Zhang, L., Mitchell, S. H., \& de Wit, H. (1999). Delay or probability discounting in a model of impulsive behavior: Effect of alcohol. Journal of the Experimental Analysis of Behavior, 71, 121-143.

Roth, R. H., Tam, S. Y., Ida, Y., Yang, J. X., \& Deutch, A. Y. (1988). Stress and the mesocorticolimbic dopamine systems ${ }^{\mathrm{a}}$. Annals of the New York Academy of Sciences, 537, 138-147.

Selim, M., \& Bradberry, C. W. (1996). Effect of ethanol on extracellular 5-HT and glutamate in 
the nucleus accumbens and prefrontal cortex: Comparison between the Lewis and Fischer 344 rat strains. Brain Research, 716, 157-164.

Simon, N. W., LaSarge, C. L., Montgomery, K. S., Williams, M. T., Mendez, I. A., Setlow, B., et al. (2010). Good things come to those who wait: Attenuated discounting of delayed rewards in aged Fischer 344 rats. Neurobiology of Aging, 31, 853-862.

Simon, N. W., Mendez, I. A., \& Setlow, B. (2007). Cocaine exposure causes long-term increases in impulsive choice. Behavioral Neuroscience, 121(3), 543-549.

Slezak, J. M., \& Anderson, K. G. (2009). Effects of variable training, signaled and unsignaled delays, and $d$-amphetamine on delay-discounting functions. Behavioural Pharmacology, 20, 424-436.

Stein, L., Wise, C. D., \& Belluzzi, J. D. (1975). Effects of benzodiazepines on central serotonergic mechanisms. Advances in Biochemical Psychopharmacology, 14, 29-44.

Suzuki, T., George, F. R., \& Meisch, R. A. (1988). Differential establishment and maintenance of oral ethanol reinforced behavior in Lewis and Fischer 344 inbred rat strains. The Journal of Pharmacology and Experimental Therapeutics, 245(1), 164-170.

Suzuki, T., Lu, M. S., Motegi, H., Yoshii, T., \& Misawa, M. (1992). Genetic differences in the development of physical dependence upon diazepam in Lewis and Fischer 344 inbred rat strains. Pharmacology, Biochemistry, and Behavior, 43, 387-393.

Suzuki, T., Otani, K., Koike, Y., \& Misawa, M. (1988). Genetic differences in preferences for morphine and codeine in Lewis and Fischer 344 inbred rat strains. Japanese Journal of Pharmacology, 47, 425-431.

Takahashi, R. N., Berton, O., Mormede, P., \& Chaouloff, F. (2001). Strain-dependent effects of diazepam and the 5-HT2B/2C receptor antagonist SB 206553 in spontaneously 
hypertensive and Lewis rats tested in the elevated plus-maze. Brazillian Journal of Medical and Biological Research, 34, 675-682.

Thiebot, M. H., Bihan, C. L., Soubrie, P., \& Simon, P. (1985). Benzodiazepines reduce the tolerance to reward delay in rats. Psychopharmacology, 86, 147-152.

Tomie, A., Aguado, A. S., Pohorecky, L. A., \& Benjamin, D. (1998). Ethanol induces impulsive-like responding in a delay-of-reward operant choice procedure: Impulsivity predicts autoshaping. Psychopharmacology, 139, 376-382.

Wade, T. R., de Wit, H., \& Richards J. B. (2000). Effects of dopaminergic drugs on delayed reward as a measure of impulsive behavior in rats. Psychopharmacology, 150, 90-101.

Weiss, F., Lorang, M. T., Bloom, F. E., \& Koob, G. F. (1993). Oral alcohol self-administration stimulates dopamine release in the rat nucleus accumbens: Genetic and motivational determinants. The Journal of Pharmacology and Experimental Therapeutics, 267, 250258.

Wilhelm, C. J., \& Mitchell, S. H. (2009). Strain differences in delay discounting using inbred rats. Genes, Brain, and Behavior, 8, 426-434.

Winstanley, C. A., Dalley, J. W., Theobald, E. H., \& Robbins, T. W. (2003). Global 5-HT depletion attenuates the ability of amphetamine to decrease impulsive choice on a delaydiscounting task in rats. Psychopharmacology, 170, 320-331.

Wogar, M. A., Bradshaw, C. M., \& Szabadi, E. (1993). Effect of lesions of the ascending 5hydroxytryptaminergic pathways on choice between delayed reinforcers. Psychopharmacology, 111, 239-243.

Woods, J. H., Katz, J. L., \& Winger, G. (1987). Abuse liability of benzodiazepines. Pharmacological Reviews, 39, 251-413. 
Yoshimoto, K., McBride, W. J., Lumeng, L., \& Li, T. K. (1992). Alcohol stimulates the release of dopamine and serotonin in the nucleus accumbens. Alcohol, 9, 17-22.

John H. 